\title{
Foxp1 regulation of neonatal vocalizations via cortical development
}

\author{
Noriyoshi Usui, ${ }^{1,2,3}$ Daniel J. Araujo, ${ }^{1,7}$ Ashwinikumar Kulkarni, ${ }^{1}$ Marissa Co, ${ }^{1}$ Jacob Ellegood, ${ }^{4}$ \\ Matthew Harper, ${ }^{1}$ Kazuya Toriumi, ${ }^{1,5}$ Jason P. Lerch, ${ }^{4,6}$ and Genevieve Konopka ${ }^{1}$ \\ ${ }^{1}$ Department of Neuroscience, University of Texas Southwestern Medical Center, Dallas, Texas 75390, USA; ${ }^{2}$ Division of \\ Development of Mental Functions, Research Center for Child Mental Development, University of Fukui, Fukui 910-1193, Japan; \\ ${ }^{3}$ Division of Developmental Higher Brain Functions, Department of Child Development, United Graduate School of Child \\ Development, Osaka University, Kanazawa University, Hamamatsu University School of Medicine, Chiba University, and \\ University of Fukui, Osaka 565-0871, Japan; ${ }^{4}$ Mouse Imaging Centre (MICe), Hospital for Sick Children, Toronto, Ontario M5S \\ 1A1, Canada; ${ }^{5}$ Project for Schizophrenia Research, Department of Psychiatry and Behavioral Sciences, Tokyo Metropolitan \\ Institute of Medical Science, Tokyo 156-8506, Japan; ${ }^{6}$ Department of Medical Biophysics, University of Toronto, Toronto, Ontario \\ M5S 1A1, Canada
}

The molecular mechanisms driving brain development at risk in autism spectrum disorders (ASDs) remain mostly unknown. Previous studies have implicated the transcription factor FOXP1 in both brain development and ASD pathophysiology. However, the specific molecular pathways both upstream of and downstream from FOXP1 are not fully understood. To elucidate the contribution of FOXP1-mediated signaling to brain development and, in particular, neocortical development, we generated forebrain-specific Foxp1 conditional knockout mice. We show that deletion of Foxp1 in the developing forebrain leads to impairments in neonatal vocalizations as well as neocortical cytoarchitectonic alterations via neuronal positioning and migration. Using a genomics approach, we identified the transcriptional networks regulated by Foxp1 in the developing neocortex and found that such networks are enriched for downstream targets involved in neurogenesis and neuronal migration. We also uncovered mechanistic insight into Foxp1 function by demonstrating that sumoylation of Foxp1 during embryonic brain development is necessary for mediating proper interactions between Foxp1 and the NuRD complex. Furthermore, we demonstrated that sumoylation of Foxp1 affects neuronal differentiation and migration in the developing neocortex. Together, these data provide critical mechanistic insights into the function of FOXP1 in the developing neocortex and may reveal molecular pathways at risk in ASD.

[Keywords: autism; behavior; neocortex; neurogenomics; neuronal migration; sumoylation]

Supplemental material is available for this article.

Received July 19, 2017; revised version accepted October 25, 2017.

FOXP1 is among the relatively few high-confidence genes with de novo mutations associated with autism spectrum disorders (ASDs) (Iossifov et al. 2014; Sanders et al. 2015; Stessman et al. 2017). Individuals with FOXP1 mutations are diagnosed with ASD and/or intellectual disability (ID) and present with global developmental delay, motor delay, and severe speech delay (Bacon and Rappold 2012). The majority of mutations in FOXP1 (deletions, translocations, missense, and nonsense mutations) (Hamdan et al. 2010; O'Roak et al. 2011; Bacon and Rappold 2012; Lozano et al. 2015; Sollis et al. 2016) results in loss of function (Sollis et al. 2016). However, detailed loss-of-function studies investigating the contributions of FOXP1 to ASD-relevant phenotypes in the developing brain have yet to be carried out.

\footnotetext{
${ }^{7}$ Present Address: Department of Molecular and Cell Biology, University of California at Berkeley, Berkeley, CA 94720, USA. Corresponding author: genevieve.konopka@utsouthwestern.edu Article published online ahead of print. Article and publication date are online at http://www.genesdev.org/cgi/doi/10.1101/gad.305037.117.
}

A few mouse models of Foxp1 have begun to elucidate the function of Foxp1, primarily in the adult brain. (Forkhead genes have a unique protein nomenclature: uppercase for primates, title case for rodents, and mixed case for all other species or mixture of species [Kaestner et al. 2000].) Adult mice with a brain-wide deletion of Foxp1 using a conditional knockout (cKO) approach (Nes-Cre; Foxp $1^{\text {flox/flox })}$ demonstrated developmental abnormalities in the striatum and hippocampus, reduced excitability of hippocampal CA1 neurons, impaired short-term memory, and ASD-like behaviors (Bacon et al. 2015). In addition, our patient-relevant haploinsufficient Foxp1 mouse model displayed increased excitability of striatal medium spiny neurons (MSNs), altered neonatal ultrasonic vocalizations (USVs), and altered gene expression of ASD-

(C) 2017 Usui et al. This article is distributed exclusively by Cold Spring Harbor Laboratory Press for the first six months after the full-issue publication date (see http://genesdev.cshlp.org/site/misc/terms.xhtml). After six months, it is available under a Creative Commons License (Attribution-NonCommercial 4.0 International), as described at http:// creativecommons.org/licenses/by-nc/4.0/. 
relevant pathways as adults (Araujo et al. 2015). Together, these studies have demonstrated the importance of Foxp1 in global brain development. However, the region-specific roles of Foxp1, in particular its role in neocortical development and function, are largely unknown.

Previous work has demonstrated that genes expressed during fetal brain development are at risk in ASDs (Willsey et al. 2013; de la Torre-Ubieta et al. 2016; Packer 2016). These findings suggest that early brain development is a critical time point for understanding the mechanisms underlying ASDs. Therefore, in this study, we characterized the behavioral and neuroanatomical phenotypes in forebrain-specific Foxp1 cKO mice (Emx1Cre; Foxp $1^{\text {flox/flox }}$ ) at early postnatal stages that are analogous to the phases of early human fetal brain development. We demonstrate impairments in USV production and the neocortical cytoarchitecture of Foxp1 cKO mice. To explore the molecular mechanisms underlying these phenotypes, we carried out RNA sequencing (RNA-seq) and identified alterations in the expression of genes involved in neuronal development, migration, and synaptic function. Moreover, we show that sumoylation of Foxp1 in the neocortex plays an essential role in neurite outgrowth and neuronal migration. Finally, we found that sumolyation regulates the recruitment of the FOXP1-NuRD complex, a potential mechanism to modify gene expression. Together, these data demonstrate a critical role for Foxp1 in the developing forebrain that may be relevant to neurodevelopmental disorders such as ASDs.

\section{Results}

\section{Forebrain expression of Foxp1 is required for vocal} communication

We generated Foxp1 cKO mice by crossing Emx1-Cre driver mice (Gorski et al. 2002) to Foxp1 $1^{\text {flox/flox }}$ mice (Feng et al. 2010) as described previously (Araujo et al. 2017). We confirmed an absence of Foxp1 expression in the postnatal neocortices and hippocampi of these animals (Fig. 1A,B). In general, Foxp1 cKO mice appeared healthy and viable, and there was no difference in their average body weight during early postnatal development compared with littermate controls (Fig. 1C). We performed behavioral analysis for USVs as an ASD-relevant phenotype at early postnatal stages on Foxp1 cKO mice and observed alterations in USVs at postnatal day $4(\mathrm{P} 4)$ and $\mathrm{P} 7$ (Fig. 1D-I). Specifically, the number of whistle calls produced was significantly lower (interaction, $P=0.0036$; age, $P<0.0001$; genotype, $P<0.0001$ ) (Fig. 1D), the percentage of calls with frequency jumps was lower (interaction, $P$ $=0.67$; age, $P=0.020$; genotype, $P=0.0016)$ (Fig. $1 \mathrm{E})$, call durations were shorter (interaction, $P=0.79$; age, $P<$ 0.0001; genotype, $P<0.0001$ ) (Fig. $1 \mathrm{~F}$ ), and the frequency range was smaller (interaction, $P=0.59$; age, $P=0.031$; genotype, $P<0.0001$ ) (Fig. 1I). However, there were no significant differences in either the mean frequency or call slope of Foxp 1 cKO mouse USVs (Fig. 1G,H). These data suggest that Foxp1 expression in the pyramidal neurons of the fore- brain is necessary for proper vocal communication at early postnatal stages.

\section{Deletion of Foxp1 in the forebrain results in structural brain changes and abnormal neocortical neuronal positioning}

To quantify any neuroanatomical phenotypes corresponding to forebrain-specific loss of Foxp1 that might be contributing to the observed behavioral phenotypes, we analyzed the brains of Foxp1 cKO mice at P7 by magnetic resonance imaging (MRI). For this assessment, we looked at relative volume differences regionally as well as voxelwise, as there was a significant decrease in the total brain volume $(-4.4 \% ; P=0.01 ; q=0.03)$ of Foxp 1 cKO mice. Assessing brain areas with robust EMX1 expression (Gorski et al. 2002), we found significant reductions in the relative volume (percentage total brain volume) of the cerebral cortex and hippocampus and an increase in the relative volume of the lateral septum (Fig. 2A,B; Supplemental Table S1). Interestingly, we also found significant changes in white matter tracts and brain regions not expected to be directly affected by Emx1-Cre expression (Fig. 2A,B; Supplemental Table S1). These results demonstrate that deletion of Foxp1 grossly impacts brain development. These data also suggest that loss of Foxp1 might alter the numbers of forebrain projection neurons, leading to alterations in white matter tract density, and affect brain development in a non-cell-autonomous manner.

Previous neuroanatomical and neuroimaging studies have shown that the neocortex is an important driver for vocalization-related motor functions, with little involvement of the hippocampus (Konopka and Roberts 2016b). Thus, we focused our subsequent studies on the neocortex in order to identify the molecular pathways that might explain the vocal abnormalities observed in Foxp1 cKO mice. We first investigated whether loss of Foxp1 alters the cytoarchitectonic structure of the neocortex by examining the expression of well-characterized neocortical layer markers. We immunostained neocortical sections from Foxp1 cKO mice at P7 for the layer 2-4 marker CUX1, the layer 5 marker CTIP2, and the layer 6 marker Foxp2. In line with our MRI data, we found that loss of Foxp1 leads to a smaller neocortex (Fig. 2C,D; Supplemental Fig. S1; Supplemental Table S1), and this decrease is caused primarily by a reduction in the size of layer 6 (Fig. 2C-F). Interestingly, the reduction in size of layer 6 does not correspond to a significant decrease in the number of layer 6 Foxp2-positive neurons (Fig. 2F), suggesting that a smaller layer 6 could be due to loss of other cell types in layer 6 (non-Foxp2-positive cells) or changes in the spacing between cells. In addition, we quantified relative thicknesses of CUX1-positive, CTIP2-positive, and Foxp2-positive layers within layers $2-4$, layer 5 , and layer 6 , respectively (Fig. 2C,E). We found that there was an increase in the relative size of layers 2-4 and again a decrease in the size of layer 6 , with no change in layer 5. By quantifying the number of cells in each layer using the same markers, we found a large increase in the number of CUX1-positive cells in layers 5 and 6 (Fig. 2F), suggesting abnormal positioning 
A

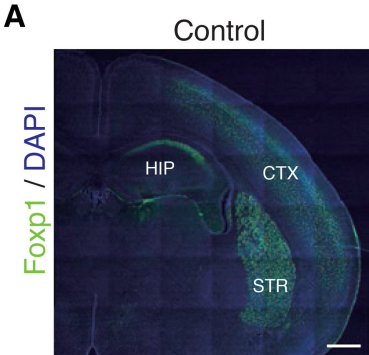

C
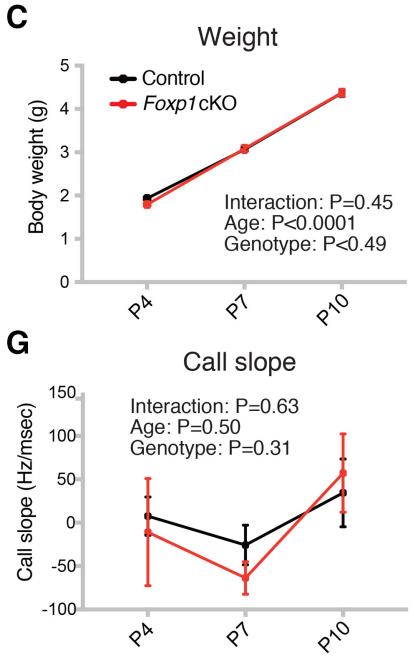

Foxp1 cKO

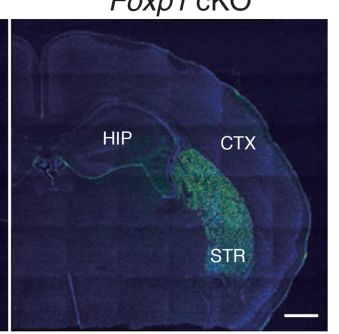

D
Whistle calls

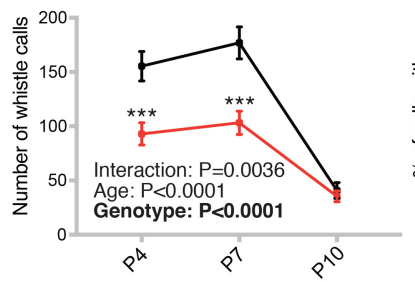

H

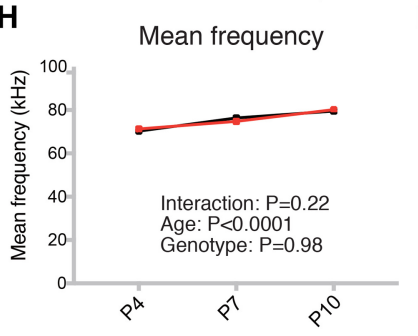

B

Mouse brain

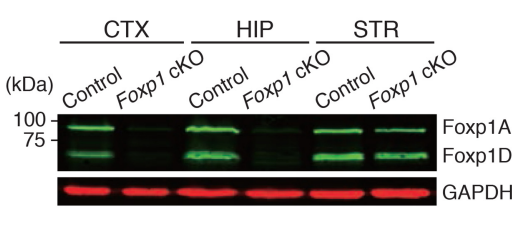

E
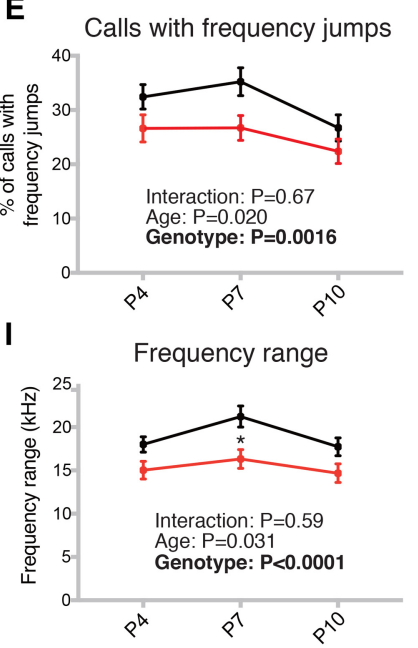
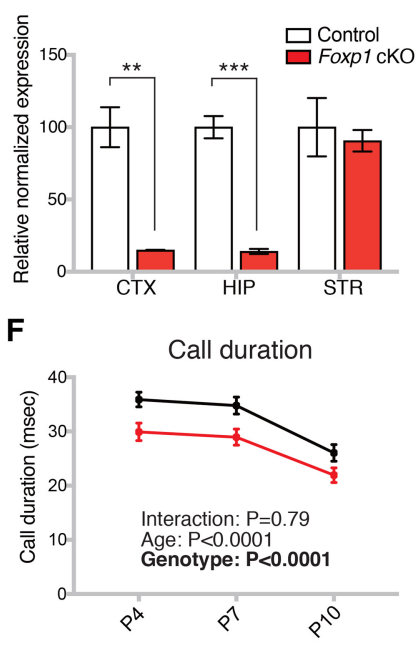

Figure 1. Foxp1 cKO mice exhibit abnormalities in vocal communication. (A) Immunostaining of Foxp1 expression in control $\left(\right.$ Foxp $\left.1^{\text {flox/flox }}\right)$ and Foxp1 cKO (Emx1-Cre; Foxp $\left.1^{\text {flox/flox }}\right)$ mice at P7. (CTX) Neocortex; (HIP) hippocampus; (STR) striatum. (B) Representative immunoblot and quantification of Foxp1 expression in each brain region of control and Foxp $1 \mathrm{cKO}$ mice at P7. Data are represented as means $\left.\left({ }_{ \pm} \mathrm{SEM}\right) .\left(^{* * *}\right) P<0.001 ;{ }^{* *}\right) P<0.01, t$-test. $n=3$ per condition. $(C)$ Mouse weight at postnatal stages. Data are represented as means $( \pm$ SEM). Interaction, $P=0.45$; age, $P<0.0001$; genotype, $P<0.49$, two-way ANOVA with a Tukey's multiple comparison test. $n=46-48$ per condition. (D-I) Analysis of USVs. $(D)$ Total number of whistle calls (USVs). Interaction, $P=0.0036$; age, $P<0.0001$; genotype, $P<$ 0.0001. (E) Percentage of calls with frequency jumps. Interaction, $P=0.67$; age, $P=0.020$; genotype, $P=0.0016$. $(F)$ Call duration. Interaction, $P=0.79$; age, $P<0.0001$; genotype, $P<0.0001$. $(G)$ Call slope. Interaction, $P=0.63$; age, $P=0.50$; genotype, $P=0.31$. $(H)$ Mean frequency. Interaction, $P=0.22$; age, $P<0.0001$; genotype, $P=0.98$. (I) Frequency range. Interaction, $P=0.59$; age, $P=0.031$; genotype, $P<0.0001$. Data are represented as means $\left({ }_{ \pm} \mathrm{SEM}\right) .\left(^{* * *}\right) P<0.001 ;\left(^{*}\right) P<0.05$, two-way ANOVA with a Tukey's multiple comparison test. $n=51-52$ per condition. Bars, $500 \mu \mathrm{m}$.

of CUX1-positive neurons in the deep layers of Foxp1 cKO mice. We also found a small but significant increase in the number of CTIP2-positive neurons in layer 5 (Fig. 2F). Together, these data suggest that forebrain-specific loss of Foxpl affects neocortical lamination and overall neocortical size, potentially through a defect in neocortical migration given that CUX1-positive neurons are mispositioned in deep layers (Supplemental Fig. S1).

\section{Identification of Foxp1-mediated signaling pathways in neocortical development}

To identify molecular pathways downstream from Foxp1 that might contribute to the observed behavioral and neuroanatomical deficits of Foxp1 cKO mice, we carried out RNA-seq in the neocortices of Foxp1 cKO mice and littermate controls at P0 and P7. At P0, we identified 591 differentially expressed genes (DEGs; 203 up-regulated and 388 down-regulated) (Fig. 3A; Supplemental Table S2). Gene ontology (GO) analysis of the DEGs identified enrichment for synaptic regulation and neuronal projection pathways among the up-regulated genes (Fig. 3B; Supplemental Table S3) and enrichment for neuronal development, proliferation, and migration pathways among the down-regulated genes at P0 (Fig. 3C; Supplemental Table S3). At P7, we identified 327 DEGs (124 up-regulated and 203 downregulated) (Fig. 3D), and up-regulated genes were enriched for neurogenesis pathways (Fig. 3E; Supplemental Table S3), while down-regulated genes were enriched for channel activity and neuronal projection pathways (Fig. 3F; Supplemental Table S3). These results suggest that expression of Foxp1 in the neocortex is important for regulating the expression of genes involved in neuronal development, migration, and synaptic function. To determine the potential disease relevance of these Foxp1-regulated DEGs, we intersected the DEGs with ASD genes derived from the SFARI gene database (see the Materials and Methods; Supplemental Table S4) and found that the intersection of each DEG list significantly overlapped with the SFARI gene list (Fig. 3G,H; Supplemental Table 
A

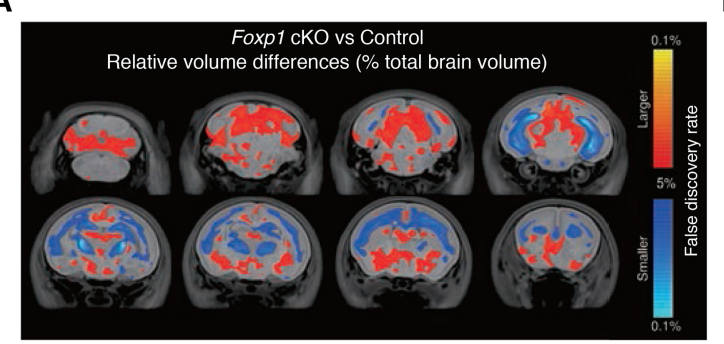

B

\begin{tabular}{|c|c|c|c|c|c|c|c|c|}
\hline \multirow[b]{2}{*}{ Brain regions } & \multicolumn{2}{|c|}{ Control } & \multicolumn{2}{|c|}{ Foxp1cKO } & \multirow[b]{2}{*}{$\%$ Diff } & \multirow[b]{2}{*}{ Effect } & \multirow[b]{2}{*}{ P-value } & \multirow[b]{2}{*}{ FDR } \\
\hline & Mean & SD & Mean & SD & & & & \\
\hline hippocampus & 4.64 & 0.12 & 4.16 & 0.10 & -10.43 & -3.89 & 8.83E-14 & $4.94 \mathrm{E}-12 * * *$ \\
\hline dentate gyrus of hippocampus & 0.67 & 0.02 & 0.62 & 0.02 & -7.58 & -2.84 & 2.02E-09 & 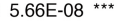 \\
\hline corpus callosum & 3.16 & 0.07 & 3.01 & 0.05 & -4.66 & -2.07 & 1.06E-07 & $1.98 \mathrm{E}-06$ \\
\hline cerebral cortex parieto temporal lobe & 20.52 & 0.32 & 19.86 & 0.35 & -3.17 & -2.01 & 4.92E-06 & $6.15 \mathrm{E}-05 * \star *$ \\
\hline cerebral cortex frontal lobe & 8.80 & 0.14 & 8.58 & 0.19 & -2.55 & -1.63 & $6.56 \mathrm{E}-04$ & $2.45 \mathrm{E}-03$ *** \\
\hline lateral olfactory tract & 0.09 & 0.00 & 0.10 & 0.00 & 4.25 & 1.37 & $1.61 \mathrm{E}-03$ & 3.74E-03 ** \\
\hline fornix & 0.19 & 0.01 & 0.20 & 0.01 & 4.52 & 1.60 & 3.43E-04 & $1.71 \mathrm{E}-03^{\star \star \star}$ \\
\hline cereb & 0.03 & 0.00 & 0.04 & 0.00 & 4.61 & 0.97 & $1.24 \mathrm{E}-02$ & $2.57 \mathrm{E}-02$ * \\
\hline poste & 0.00 & 0.00 & 0.01 & 0.00 & 5.21 & 2.01 & 3.80E-04 & 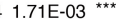 \\
\hline lateral septum & 1.06 & 0.03 & 1.11 & 0.03 & 5.40 & 2.05 & 5.49E-06 & $6.15 \mathrm{E}-05^{\star \star \star}$ \\
\hline
\end{tabular}

C

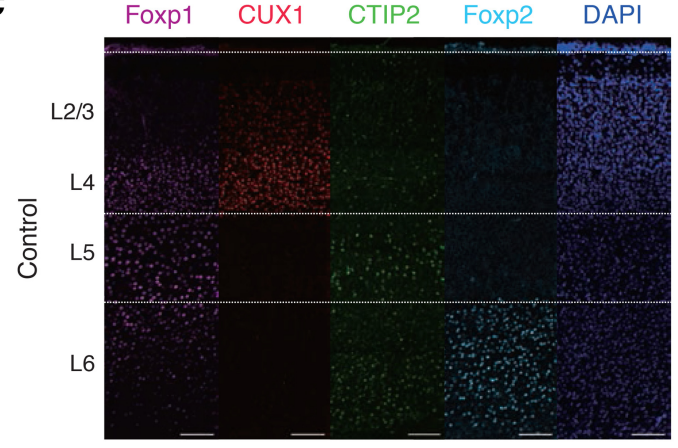

D

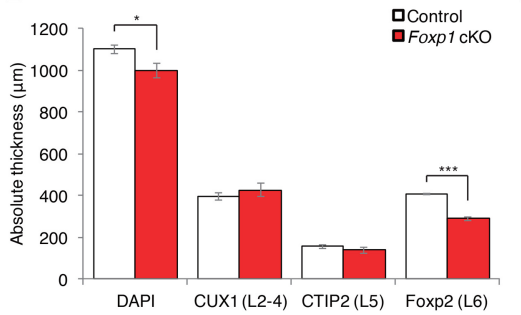

E

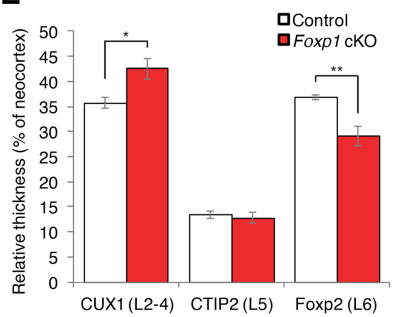

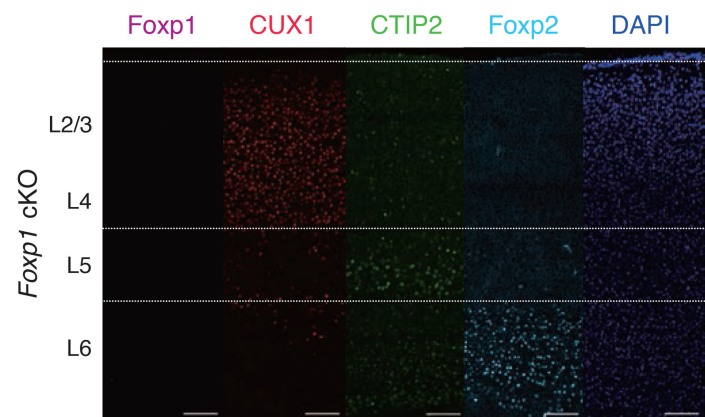

F

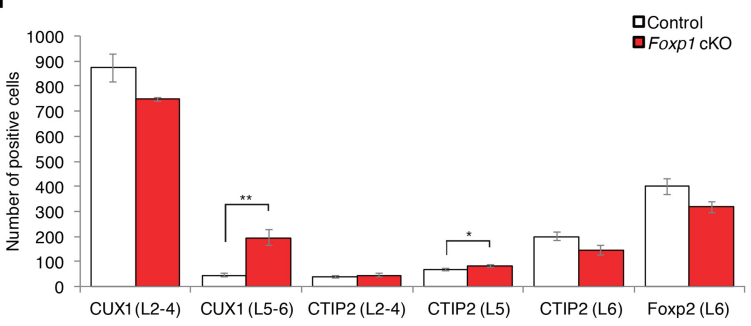

Figure 2. Foxp1 cKO mice have altered brain structure and neocortical cytoarchitecture. $(A)$ Representative coronal brain slices of magnetic resonance images highlighting in each slice the relative volume differences in areas that are larger (in red) or smaller (in blue) in Foxp1 cKO mice compared with controls. False discovery rate $(\mathrm{FDR})<0.05 .(B)$ Quantification table for the brain regions with robust EMX1 expression that demonstrate significantly altered relative volumes in Foxp $1 \mathrm{cKO}$ mice at P7. Data are represented as means $( \pm \mathrm{SD}) .\left({ }^{* * *}\right) q<$ $0.005 ;\left(^{* *}\right) q<0.01 ;\left(^{*}\right) q<0.05$, FDR. $n=15-18$ per condition. $(C)$ Fluorescent images of CUX1 (layers 2-4), CTIP2 (layer 5), and Foxp2 (layer 6) immunostaining in the neocortices of coronal sections of control and Foxp1 cKO mice at P7. (D-F) Quantifications of absolute thickness (DAPI, $P=0.038$; CUX1 [L2-4], $P=0.41$; CTIP2 [L5], $P=0.32$; Foxp2 [L6], $P<0.0001)(D)$, relative thickness (CUX1 [L2-4], $P=0.019$; CTIP2 [L5], $P=0.59$; Foxp2 [L6], $P=0.0028)(E)$, and number of positive cells (CUX1 [L2-4], $P=0.09$; CUX1 [L5-6], $P=0.0013$; CTIP2 [L2-4], $P=0.63$; CTIP2 [L5], $P=0.031$; CTIP2 [L6], $P=0.062$; Foxp2 [L6], $P=0.080)(F)$ in the mouse neocortex at P7. Data are represented as means ${ }_{ \pm}$SEM). $\left({ }^{* * *}\right) P<0.001 ;\left({ }^{* *}\right) P<0.01 ;\left(^{*}\right) P<0.05, t$-test. $n=4-5$ per condition. Bars, $100 \mu \mathrm{m}$.

S4). We identified four up-regulated (Akap9, Aldh1a3, Grm1, and Smarca2) and seven down-regulated (Brinp1, Diaph3, Foxp1, Galnt14, Lpl, Snap25, and Zfp804a) genes that were altered at both time points and overlapped with the ASD genes. To further prioritize specific genes for relevance to ASDs, we performed weighted gene correlation network analysis (WGCNA) (Supplemental Table S5; Supplemental Fig. S2; Langfelder and Horvath 2008). The "pink" module was most positively associated with genotype, in particular at the P0 time point. This module contained ASD genes such as Erbin, Fbn1, Gas2, and Wac among the most highly interconnected genes (Supplemental Fig. S2C). In addition, CNEP1R1, the human ortholog of another "hub" gene, Cnep1r1, is within 16q11.2-q12.1, a locus associated with ASDs, ID, and developmental delay when deleted or duplicated (Weiss et al. 2008; Kaminsky et al. 2011). Together, these results indicate that Foxp1 regulates gene expression pathways in the developing mammalian brain that are at risk in ASD pathophysiology.

\section{Foxp1 is sumoylated during neocortical development}

The behavioral, neuroanatomical, and genomic analyses of Foxp1 cKO mice suggest that Foxp1 plays an essential role in neurodevelopment; however, the molecular mechanisms underlying the involvement of Foxp1 in neuronal development remain uncharacterized. To answer this question, we examined developmental expression of Foxp1 in the brain. As we had previously observed sumoylation of the related transcription factor Foxp2 to be important for neuronal development (Usui et al. 2017), we examined whether Foxp1 was also sumoylated during brain development. We found that Foxp1 is highly 
A

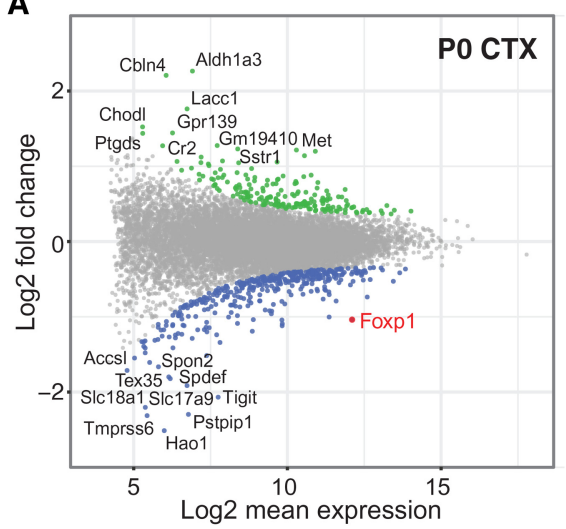

D

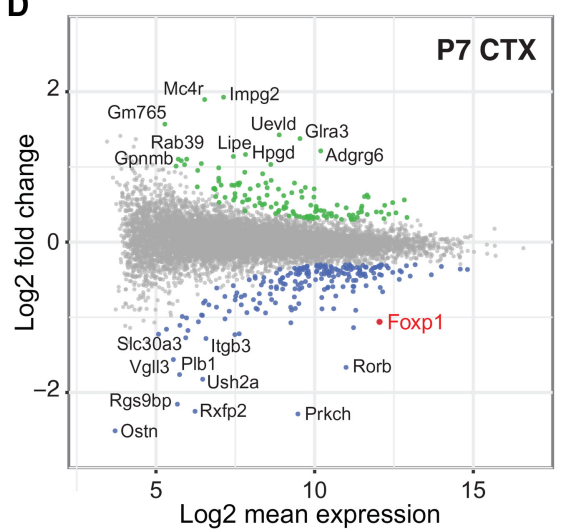

B

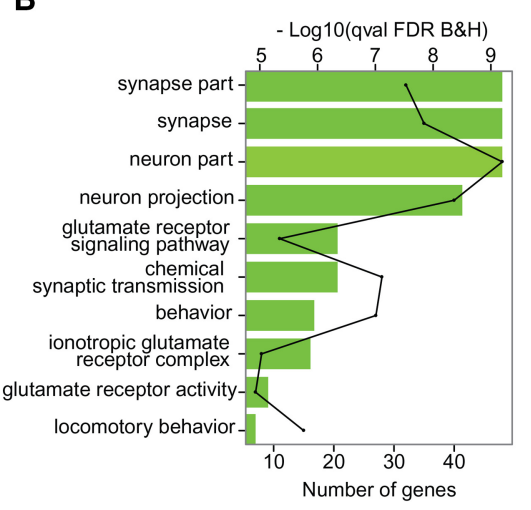

E

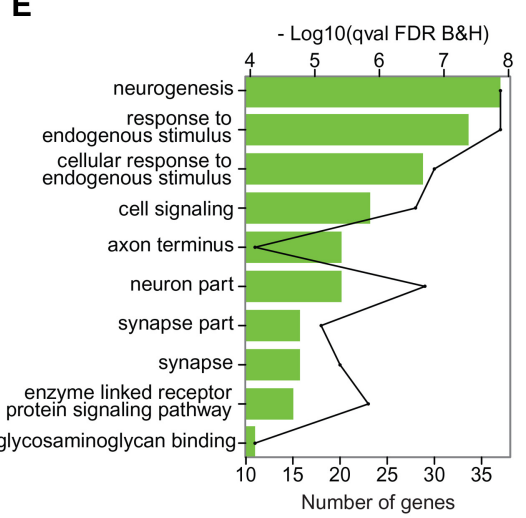

C

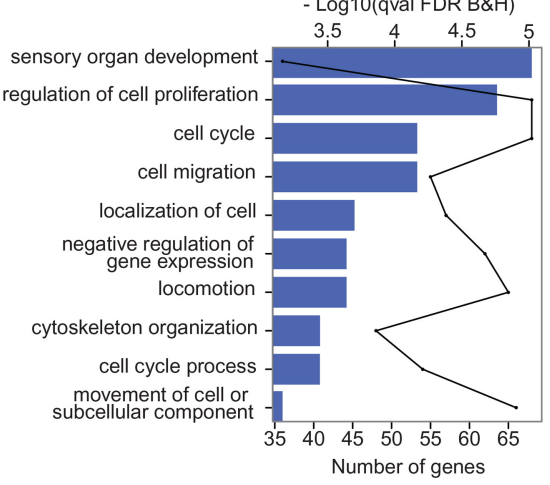

F

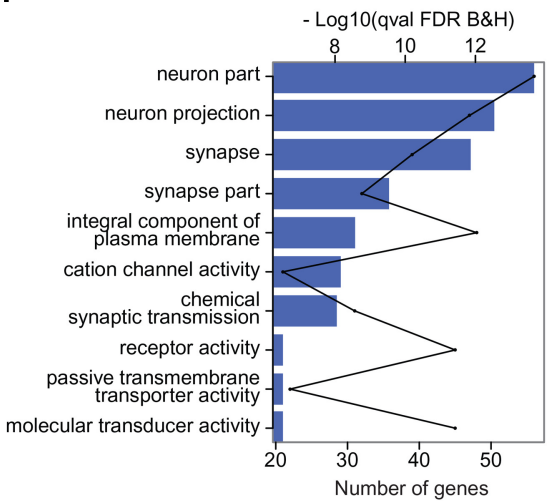

G

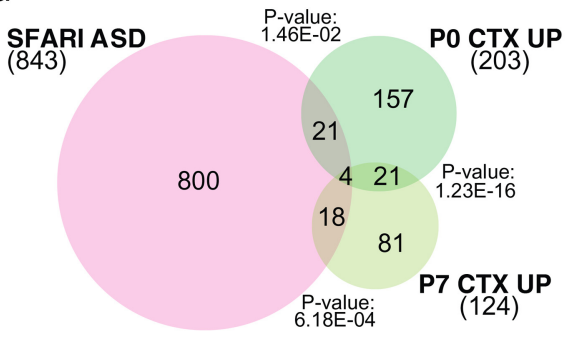

H

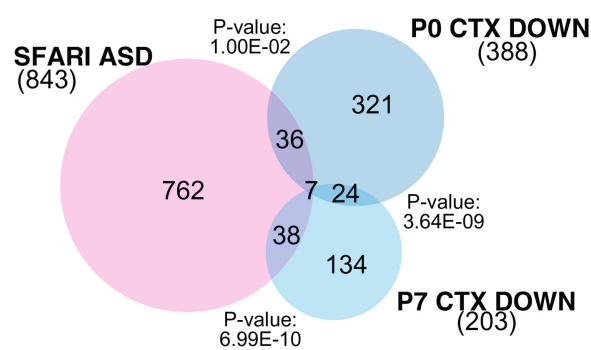

Figure 3. Gene expression changes with loss of Foxp1 in the neocortex. (A) MA plot of DEGs in the neocortex at P0. Absolute $\log _{2}$ fold change $<0.3$. FDR $<0.005$. Two-hundred-three genes were up-regulated (green) and 388 genes were down-regulated (blue) in the neocortex at P0. $(B, C) \mathrm{GO}$ analyses of up-regulated $(B)$ and down-regulated $(C)$ DEGs at P0. $(D)$ MA plot of DEGs in the neocortex at P7. One-hundredtwenty-four genes were up-regulated (green) and 203 genes were down-regulated (blue) in the neocortex at P7. (E, $F$ ) GO analyses of up-regulated $(E)$ and down-regulated $(F)$ DEGs at P7. $(G, H)$ Venn diagrams of up-regulated $(G)$ and down-regulated $(H)$ DEGs overlapping with SFARI ASD genes. (CTX) Neocortex; (UP) up-regulated genes; (DOWN) down-regulated genes.

sumoylated in the embryonic mouse neocortex in the presence of $N$-ethylmaleimide (NEM), a sumoylation stabilizer (Fig. 4A), during a developmental window corresponding to the timing of neuronal migration in this region (Molyneaux et al. 2007; Greig et al. 2013). We confirmed the endogenous sumoylation of Foxp1 in the mouse neocortex at P0 via coimmunoprecipitation of SUMO-1 and Foxp1 (Fig. 4B). We carried out in silico analysis and identified a consensus sumoylation site $(\psi \mathrm{KXE})$ at K636 (K664 in mice) of FOXP1 (Fig. 4C; Supplemental Table S6). The conservation of this site across species suggests that it plays an important role in FOXP1 function
(Fig. 4D). Next, we directly tested the formation of sumoylated FOXP1 using NEM. When we expressed Flag-tagged wild-type FOXP1 in the presence of NEM, we observed a higher-molecular-weight band. This band is recognized by either a FOXP1 or SUMO-1 antibody in lysates that have undergone immunoprecipitation with an antibody recognizing Flag. The intensity of this higher-molecularweight band was decreased by hydrogen peroxide $\left(\mathrm{H}_{2} \mathrm{O}_{2}\right)$ treatment (a mechanism for reversible inhibition of SUMO-conjugating enzymes) (Bossis and Melchior 2006) or ginkgolic acid treatment (a sumoylation-specific inhibitor) (Fig. 4E; Fukuda et al. 2009). Together, these data 
Usui et al.

A

Mouse neocortex

B

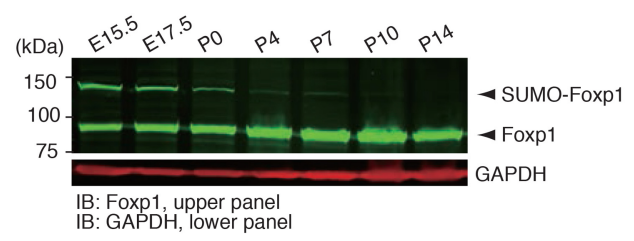

C
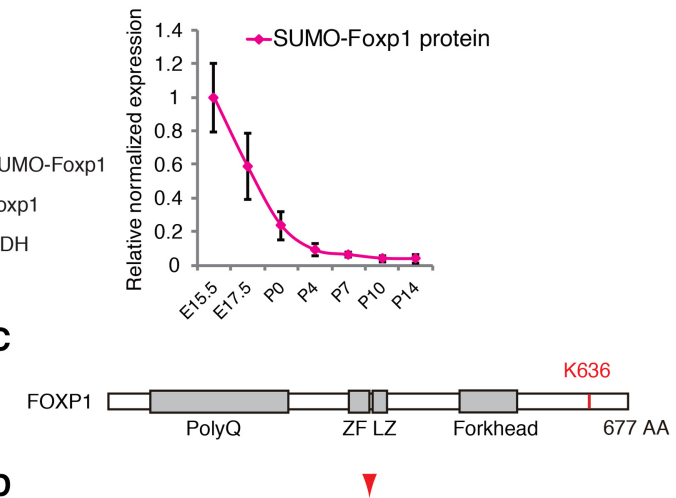

Homo sapiens Pan troglodytes Pongo abelii Gallus gallus Rattus norvegicus Mus musculus
PMQAVHPVHVKEEPLDPEEAE PMQAVHPVHV KEEPLDPEEAE PMQAVHPVHV KEEPLDPEEAE PMQAVHPVHVKEEPLDPEEAE PMQAVHPIHV KEEPLDPEEAE PMQAVHPIHV KEEPLDPEEAE
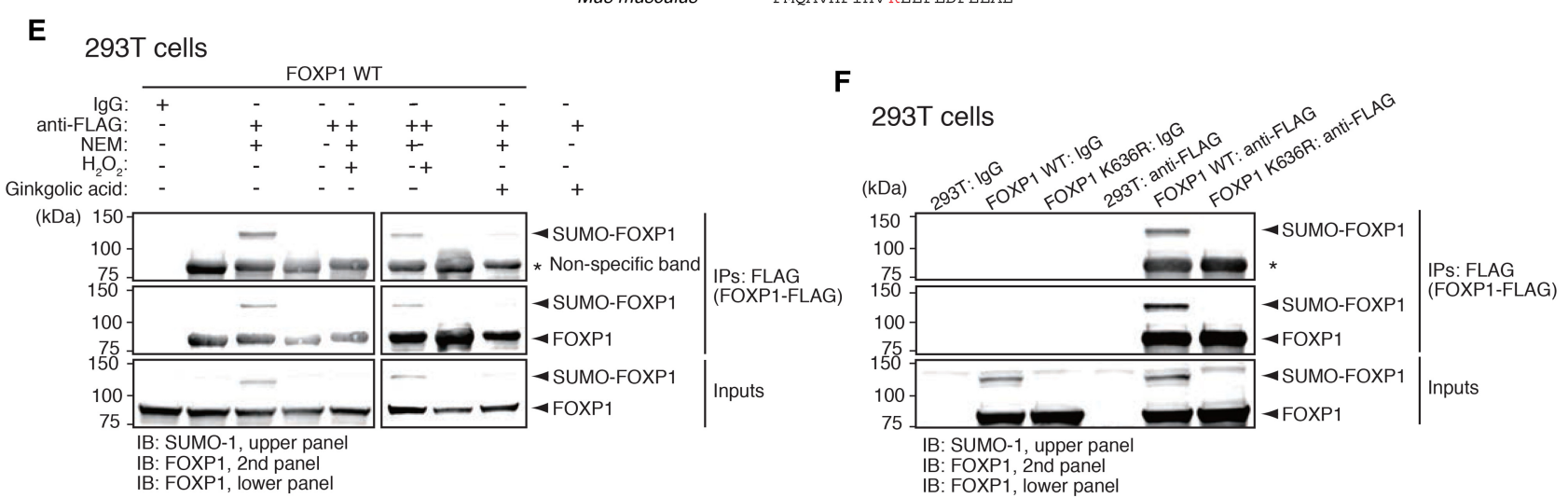

Figure 4. Sumoylation of FOXP1. (A) Representative immunoblotting of Foxp1 in the mouse neocortex during development. (Right panel) Quantification of SUMO-Foxp1 immunoblotting. Immunoblots were first normalized to glyceraldehyde-3-phosphate dehydrogenase (GAPDH) at each time point and then subsequently normalized to nonsumoylated Foxp1 levels at embryonic day 15.5 (E15.5). Data are represented as means $( \pm S E M) . n=3$ per condition. $(B)$ Endogenous coimmunoprecipitation of Foxp1 and SUMO-1 in the mouse neocortex at P0. (C) Schematic of FOXP1 protein showing the location of K636. (PolyQ) Polyglutamine motif; (ZF) zinc finger; (LZ) leucine zipper. (D) K636 is conserved across species. (E) Immunoblotting for Flag-tagged FOXP1 in $293 \mathrm{~T}$ cells. Lysates were treated with $1 \mathrm{mM} \mathrm{H}_{2} \mathrm{O}_{2}$ for $1 \mathrm{~h}$ (left panel) or $100 \mu \mathrm{M}$ ginkgolic acid for $6 \mathrm{~h}$ (right panel) in the presence or absence of NEM. (FOXP1 WT) Flag-tagged wild-type FOXP1. The asterisk indicates a nonspecific band of the SUMO-1 antibody. $(F)$ Immunoblot of immunoprecipitated wild-type Flag-tagged FOXP1 or Flag-tagged FOXP1 KR.

indicate that this higher-molecular-weight band represents a sumoylated form of FOXP1. To confirm the responsible sumoylation site, we made a mutation of lysine to arginine at K636 in FOXP1 (FOXP1 KR [FOXP1 ${ }^{\mathrm{K} 636 \mathrm{R}}$ ]) (Fig. 4C). The high-molecular-weight band was not observed with this mutation (Fig. $4 \mathrm{~F}$ ), indicating that K636 is the site of FOXP1 sumoylation.

\section{FOXP1 is sumoylated by SUMO-1/2 and PIAS E3 SUMO ligases}

To characterize the process of FOXP1 sumoylation, we investigated the interactions of FOXP1 with SUMO proteins and determined that FOXP1 is sumoylated by both SUMO-1 and SUMO-2/3 in 293T cells (Supplemental Fig. S3A,B). SUMO-2 and SUMO-3 are $97 \%$ homologous and therefore are indistinguishable (Geiss-Friedlander and Melchior 2007; Hasegawa et al. 2014). Furthermore, we identified PIAS2 and PIAS3 as potential E3 SUMO ligases (Sharrocks 2006) of FOXP1 (Supplemental Fig. S3C, D). We observed colocalization of Foxp1 with SUMO-1, SUMO-2/3, PIAS2, and PIAS3 in the cortical plate of the mouse neocortex at embryonic day 18 (E18), when Foxp1 is sumoylated (Supplemental Fig. S3E). We also observed colocalization of Foxp1 with other PIAS family proteins (PIAS1 and PIAS4) in the mouse neocortex at E18 (data not shown). These data suggest that FOXP1 is sumoylated by SUMO-1/2 proteins and PIAS family E3 SUMO ligases during embryonic time points in the neocortex.

\section{Sumoylation of FOXP1 accelerates neurite outgrowth}

Previous studies have shown that Foxp1 regulates neuronal morphogenesis (Bacon et al. 2015; Li et al. 2015), and 
sumoylation of FOXP2, a paralog of FOXP1, regulates neurite length and arborization of Purkinje cells (Usui et al. 2017). Thus, we assessed the role of FOXP1 sumoylation in neuronal morphogenesis. We measured neurite outgrowth in neocortical mouse neural progenitors (mNPs) with expression of wild-type FOXP1, FOXP1 ${ }^{\text {K636R }}$ (FOXP1 KR), or GFP control and found that wild-type FOXP1 significantly promoted the length of microtubule-associated protein 2 (MAP2)-positive mature neurites in mNPs (Fig. 5A,B). In contrast, FOXP1 KR was unable to promote neurite outgrowth as effectively (Fig. 5A,B).

\section{Sumoylation of Foxp1 controls neocortical neuronal} migration

Since we observed alterations in brain structure and neuronal positioning with loss of Foxp1 (Fig. 2), we assessed whether Foxp1 sumoylation in vivo affects neuronal migration. We carried out in utero electroporation (IUE) in Foxp1 cKO mice at E14.5 to target layer $2 / 3$ neurons (Baek et al. 2015) and then analyzed neuronal migration at E18.5. All animals received a GFP plasmid to track migration together with a rescue Foxp1 expression plasmid containing either wild-type FOXP1 (wild-type rescue) or
A Mouse neocortical neurons
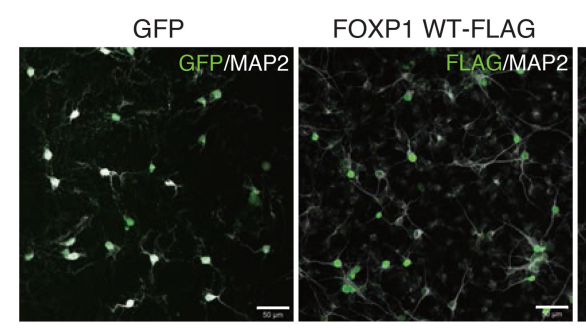

C

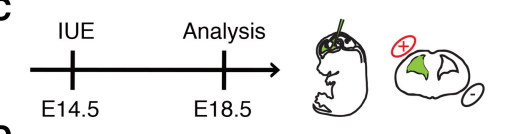

D

Mouse neocortex

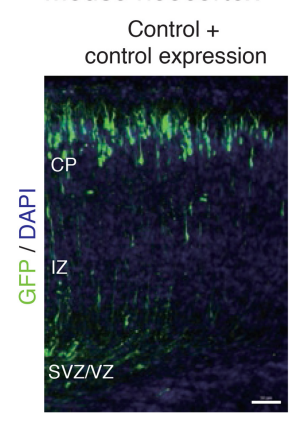

F Human neurons GFP

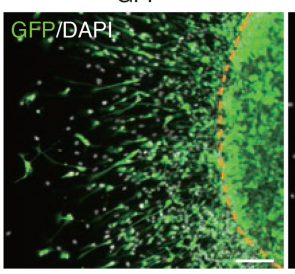

Foxp1 cKO + control expression
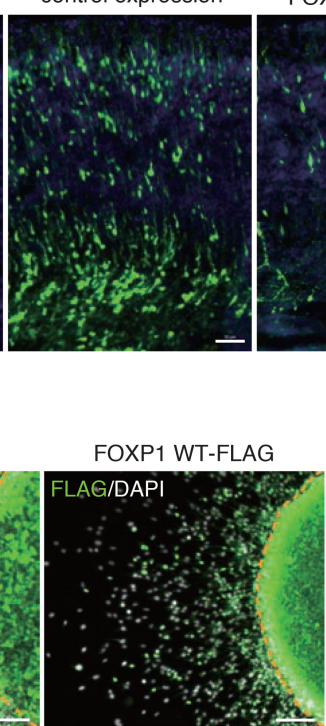

FOXP1 WT-FLAG
FOXP1 KR-FLAG
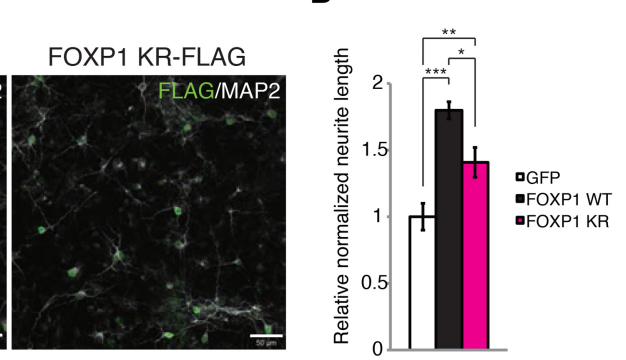

B

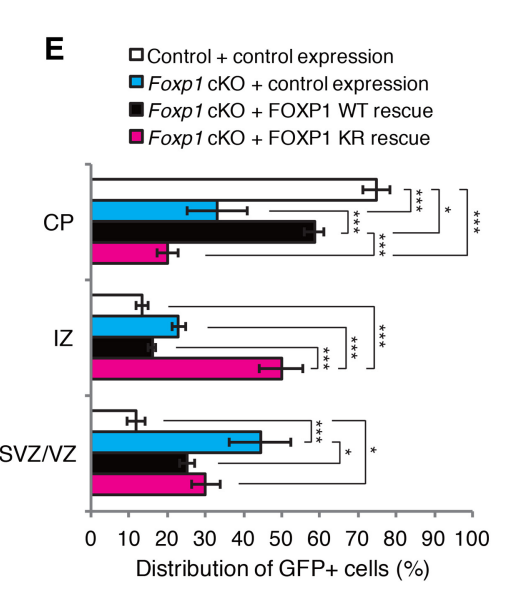

H

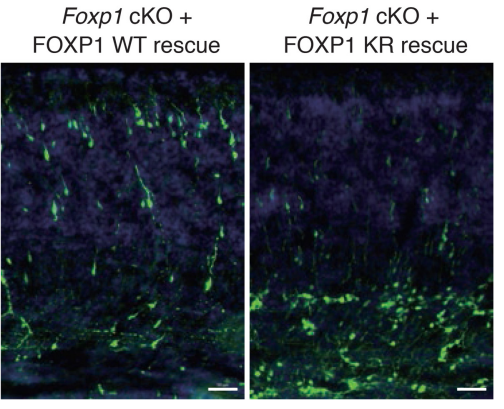

G
FOXP1 KR-FLAG

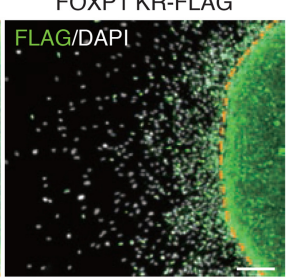

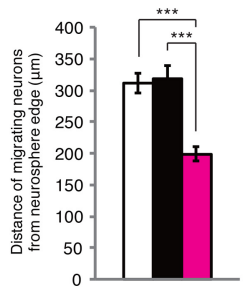

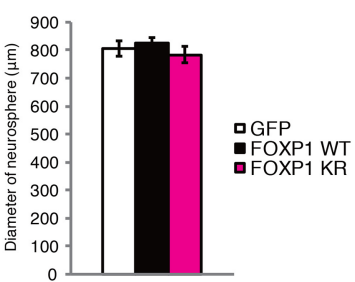

Figure 5. Sumoylation of Foxp1 is required for neurite outgrowth and neuronal migration. $(A, B)$ Immunocytochemistry $(A)$ and quantification $(B)$ of MAP2-positive neurites in differentiated mouse neocortical progenitors. Data are represented as means $( \pm S E M)$. $\left({ }^{* * *}\right) P<$ $0.001 ;\left(^{* *}\right) P<0.01 ;\left(^{*}\right) P<0.05$, one-way ANOVA with a Tukey's multiple comparison test. $n=60$ cells per condition from four independent experiments. $(C)$ Schematic and time line of in utero electroporation (IUE) in the developing neocortices of Foxp1 cKO mice. $(D)$ Immunohistochemistry of electroporated a mouse neocortex in coronal sections at E18. (E) Quantification of electroporated GFP-positive cells in a mouse neocortex at E18. (CP) Cortical plate; (IZ) intermediate zone; (SVZ/VZ) subventricular zone/ventricular zone. Data are represented as means $\left({ }_{ \pm}\right.$SEM). $\left(^{* * *}\right) P<0.001 ;\left(^{*}\right) P<0.05$, one-way ANOVA with a Tukey's multiple comparison test. $n=3-4$ per condition. $(F)$ Immunocytochemistry of neurospheres generated from human neural progenitors (hNPs). The dotted line indicates the edge of the neurosphere. $(G)$ Quantification of the distance of GFP- or Flag-positive cells from the edge of the neurospheres. Data are represented as means $( \pm$

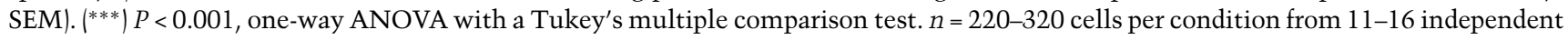
experiments. $(H)$ Quantification of the neurosphere diameters. Data are represented as means ( $\left.{ }_{ \pm} \mathrm{SEM}\right)$. Bars: $A, E, 50 \mu \mathrm{m} ; F, 100 \mu \mathrm{m}$. 
a nonsumoylated form of FOXP1 (KR rescue) (Fig. 5C). We observed abnormal migration in the neocortices of Foxp1 cKO mice, as evidenced by a significantly decreased number of cortical plate neurons and an increased number of subventricular zone/ventricular zone (SVZ/VZ) neurons (Fig. 5D,E). This migration phenotype in Foxp1 cKO mice was rescued with wild-type FOXP1 (Fig. 5D,E) but not FOXP1 KR (Fig. 5D,E), indicating that Foxp1 exerts its role in cortical neuronal migration via sumoylation.

\section{Sumoylation of FOXP1 is required for migration of human neurons in vitro}

We next investigated whether sumoylation of FOXP1 in neuronal migration could be translated to human neuronal development. We therefore used human neural progenitors (hNPs), an established in vitro model system for human neuronal development (Konopka et al. 2012; Stein et al. 2014). We created neurospheres from hNPs; transduced the cells with lentiviruses expressing wild-type FOXP1, FOXP1 KR, or GFP as a control; induced neuronal differentiation; and then quantified the migration distance of cells from the edge of the neurospheres, similar to a previous study (Baek et al. 2015). Cells from neurospheres expressing wild-type FOXP1 migrated distances similar to those of GFP-expressing controls (Fig. 5F,G). However, we observed a reduction in the migration distance of cells expressing FOXP1 KR compared with wild-type FOXP1 or GFP control cells (Fig. 5F,G). We also quantified the diameter of the neurospheres to assess any issues with sphere formation, but there was no difference among conditions (Fig. $5 \mathrm{H}$ ). These results indicate that FOXP1 sumoylation plays an important role in human neuronal migration in vitro. However, it should be noted that these in vitro results are challenging to interpret with respect to in vivo behavior and are not akin to movement along radial glia in the in vivo environment.

\section{Sumoylation of FOXP1 does not affect known protein- protein interactions}

To investigate the functional consequences of FOXP1 sumoylation, we assessed whether FOXP1 ${ }^{\mathrm{K} 636}$ affects dimerization with other FOXP family proteins or the corepressor CTBP (Li et al. 2004; Usui et al. 2017), as sumoylation often affects protein-protein interactions (Gill 2003). We overexpressed Flag-tagged wild-type FOXP1 or FOXP1 KR with each partner in $293 \mathrm{~T}$ cells and found that FOXP1 sumoylation does not affect heterodimerization or homodimerization with FOXP2, FOXP4, FOXP1, FOXP1 KR, or nonsumoylated FOXP2 ${ }^{\text {K674R }}$ (FOXP2 KR) (Usui et al. 2017) or interaction with CTBP (Supplemental Fig. S4). These data indicate that sumoylation does not affect interactions of FOXP1 with some of its major interactors.

\section{Sumoylation switches FOXP1-NuRD complex recruitment}

FOXP1/2/4 proteins have been shown to interact with GATAD2B (also known as p66 3 ), a component of the
NuRD chromatin remodeling complex, to repress transcription of their target genes (Fig. 6A; Chokas et al. 2010). The NuRD complex is formed by CHD3/4/5, HDAC1/2, MBD1/2/3, MTA1/2/3, RBBP4/7, and GATAD2A/B and interacts with a number of transcription factors to control gene expression (Chen and Dent 2014; Basta and Rauchman 2015). Interestingly, HDAC1/2 and $\mathrm{CHD} 3 / 4 / 5$ regulate neuronal proliferation and migration in the mouse neocortex (Montgomery et al. 2009; Nott et al. 2013; Nitarska et al. 2016). Therefore, we examined whether sumoylation affects FOXP1-NuRD complex formation. We first confirmed that HDAC $1 / 2$ interacts with wild-type FOXP1 as reported previously (Fig. 6B,C; Chokas et al. 2010). Remarkably, we found that the interaction of FOXP1 KR with HDAC1/2 was stronger relative to wildtype FOXP1 (Fig. 6B-D), indicating that the interaction of FOXP1 with $\mathrm{HDAC} 1 / 2$ is regulated by sumoylation. We next examined the endogenous interaction of FOXP1 with the NuRD complex using Flag-tagged FOXP1 in $293 \mathrm{~T}$ cells and pulled down major components of the NuRD complex with an anti-Flag antibody. We confirmed that $\mathrm{HDAC} 1 / 2$ were strongly immunoprecipitated by FOXP1 KR compared with wild type (Fig. 6E). We also found that MTA1/2 similarly demonstrated increased immunoprecipitation with FOXP1 KR compared with wild type (Fig. 6E); however, we did not observe a difference in GATAD2B immunoprecipitation (Fig. 6E). These data demonstrate that sumoylation regulates the affinity of FOXP1 for major components of the NuRD complex, potentially blocking the physical interaction between FOXP1 and this complex or acting as a "switch" for the disassociation of FOXP1 from this complex.

\section{Discussion}

In this study, we demonstrate that forebrain-specific Foxp1 cKO mice exhibit impaired vocal communication in early postnatal stages. We identified neuroanatomical changes with loss of Foxp1, such as a reduction in neocortical size and abnormal positioning of neurons in the deep layers of the postnatal mouse neocortex. To identify the molecular mechanisms underlying these behavioral and neuroanatomical abnormalities, we carried out RNA-seq and uncovered signaling networks regulated by Foxp1 involved in important developmental processes such as neurogenesis, neuronal migration, and synaptogenesis. Based on these phenotypes, we further investigated the developmental expression of Foxpl and demonstrated that sumoylation of Foxp 1 in the embryonic mouse neocortex controls neuronal migration. This sumoylation acts as a key for switching the molecular function of FOXP1 through alterations in the recruitment of the NuRD complex.

\section{The relevance of Foxp1 function to neurodevelopmental disorders}

Recent work has placed FOXP1 among the highest-ranked ASD candidate genes (O'Roak et al. 2011; Talkowski et al. 2012; Iossifov et al. 2014; Sanders et al. 2015). ASD is 
A FOXP1

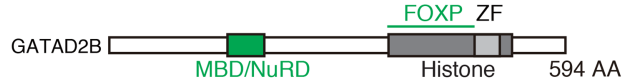

B 293T cells

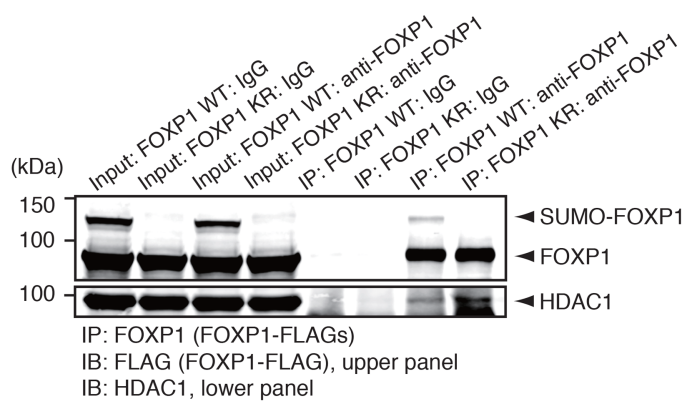

D

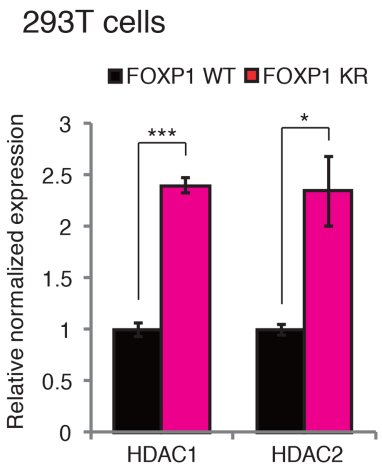

C 293T cells

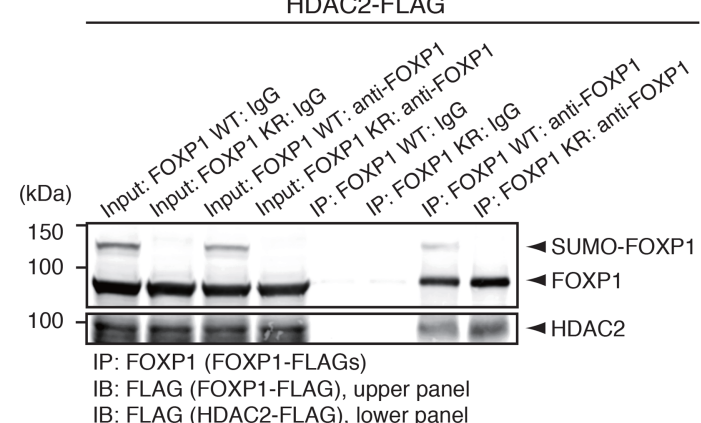

E

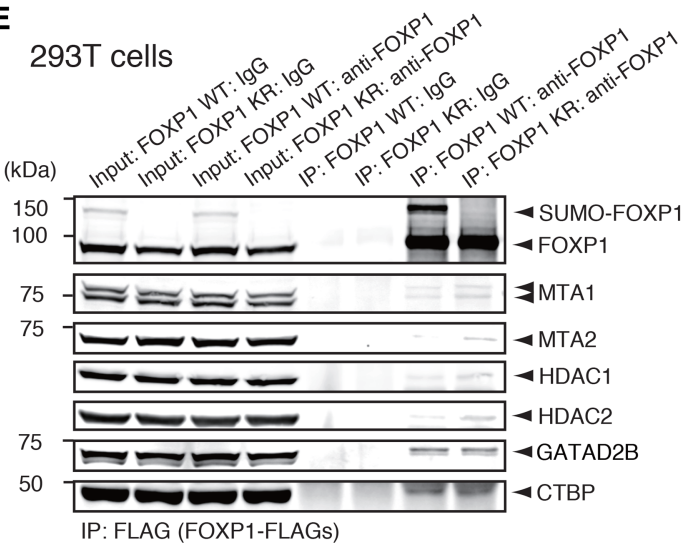

Figure 6. Sumoylation switches FOXP1-NuRD complex recruitment. (A) Schematic of FOXP1 and GATAD2B (p66ß) proteins showing each binding location. (PolyQ) polyglutamine motif; (ZF) zinc finger; (LZ) leucine zipper; (CTBP) CTBP protein-binding domain; (GATAD2B) GATAD2B-binding domain; (MBD/NuRD) MBD/NuRD-binding domain; (FOXP) FOXP-binding domain; (Histone) histone-binding domain. $(B, C)$ Coimmunoprecipitations of Flag-tagged FOXP1 and Flag-tagged HDAC1 $(B)$ or Flag-tagged HDAC2 $(C)$ in 293T cells. $(D)$ Quantification of amounts of immunoprecipitated HDACs with wild-type FOXP1 or FOXP1 KR. Data are represented as means $\left( \pm\right.$ SEM). $\left(^{* * *}\right) P<0.001 ;\left(^{*}\right) P<0.05, t$-test. $n=3$ per condition. $(E)$ Coimmunoprecipitations of Flag-tagged FOXP1 and endogenously expressed NuRD complex components in $293 \mathrm{~T}$ cells.

characterized by impairments in social communication, repetitive behaviors, and restricted interests. To elucidate a role for FOXP1 in brain development relevant to ASD, we characterized the phenotypes of forebrain-specific Foxp1 cKO mice at early postnatal stages, which are roughly equivalent to time points at risk in ASD (Willsey et al. 2013; de la Torre-Ubieta et al. 2016; Packer 2016).

We found alterations in USVs with forebrain loss of Foxp1, a phenotype relevant to ASD. Interestingly, we had demonstrated previously a reduction in USVs of Foxp1 heterozygous $\left(F_{0 x p 1^{+-}}\right)$mouse pups (Araujo et al. 2015), and the USV parameters are differentially affected between that and the present study. The changes in numbers of whistle calls (Fig. 1D), the percentage of calls with frequency jumps (Fig. 1E), call durations (Fig. 1F), and the frequency range (Fig. 1I) in Foxp1 cKO mice are phenotypes similar to those that we found in Foxp $1^{+/-}$mice. Neither call duration nor percentage of calls with frequency jumps was significantly different in Foxp $1^{+/-}$mice, whereas mean frequency and call slope were significantly different in Foxp $1^{+/}$mice but not in Foxp1 cKO mice (Fig. $1 \mathrm{G}, \mathrm{H} ;$ Araujo et al. 2015). These results suggest that Foxp1 might regulate vocal communication through multiple circuits. We found a significant reduction in the neocortical volume of Foxp1 cKO mice at P7 by MRI (Fig. 2A,B; Supplemental Table S1), underscoring the importance of neocortical function in USVs.

Studies of the language-associated gene Srpx2 have shown that modulation of synaptogenesis in the neocortex contributes to proper USV production (Sia et al. 2013). In addition, knockout mice of the Foxp1 target Cntnap2 (Vernes et al. 2008; O'Roak et al. 2011) also display reduced USVs and abnormal neuronal positioning of CUX1-positive neurons in the neocortex (Penagarikano et al. 2011). Corticostriatal circuits are associated with vocal communication in songbirds, mice, and humans (Langen et al. 2011; Murugan et al. 2013; Kalueff et al. 2016; Konopka and Roberts 2016a,b) and are frequently 
disrupted in ASD (Delmonte et al. 2013; Shepherd 2013; Kuo and Liu 2017). Moreover, the striatum has been established as a critical region for vocal production (Fisher and Scharff 2009; Frohlich et al. 2017), and our previous data have suggested important roles for Foxp1 expression in the striatum (Araujo et al. 2015) and Foxp2 expression in the cerebellum (Usui et al. 2017) for USV production. A circuit connecting projection neurons from the motor cortex to the dorsal striatum has been proposed as important for USVs in mice (Arriaga et al. 2012; Arriaga and Jarvis 2013). In contrast, many USV parameters are not affected in adult mice with developmental ablation of the neocortex (Hammerschmidt et al. 2015), suggesting that the striatum regulates the generation of calls, while the neocortex integrates sensory information important for determining when it is proper to produce calls. This idea is consistent with the hypothesis that postnatal USVs are produced in response to sensory cues upon separation from the nest (Scattoni et al. 2009; Portfors and Perkel 2014). Thus, our data suggest that neocortical expression of Foxp1, particularly in neurons involved in corticostriatal circuits, might be critical for regulating vocal communication relevant to ASD.

\section{Role of Foxp1 in brain development}

Foxp1 is highly expressed in the developing neocortex and hippocampus (Ferland et al. 2003; Kang et al. 2011). Embryonic time points are a dynamic stage of brain development, especially with regard to progenitor proliferation, neuronal specification and differentiation, and circuit formation. In this study, we demonstrate that Foxp1 is essential for normal neocortical development by identifying significant reductions in the volumes of the neocortex and hippocampus where Foxpl is highly expressed (Fig. 2A,B; Supplemental Table S1) in addition to impaired neuronal migration in the neocortex with loss of Foxp1 (Fig. $5 \mathrm{D}, \mathrm{E})$. Quantification of neocortical laminar markers also supports impairment in neuronal migration, with upper layer cell types being overrepresented in lower layers (Fig. 2C-F). Gene expression profiling in the neocortices of Foxp1 cKO mice uncovered altered signaling pathways involved in neurogenesis, neuronal migration, and synaptic function (Fig. 3).

Focusing on the handful of genes identified from the RNA-seq data that are consistently regulated by Foxp1 at both P0 and P7 (Fig. 3) further underscores the important role for Foxp1-mediated signaling pathways in brain development and the behavioral phenotypes that we observed. For example, Brinp1 knockout mice show social interaction and USV deficits, hyperactivity, and impaired short-term memory (Berkowicz et al. 2016), phenotypes reminiscent of Foxp $1 \mathrm{cKO}$ mouse phenotypes. Interestingly, the expression of neuronal migration genes Astn1 and Astn2 (Wilson et al. 2010) is up-regulated in Brinp1 knockout mice (Berkowicz et al. 2016), and Astn1 demonstrates an increase in the Foxp1 cKO neocortex at P0. Another example is DIAPH3, which is involved in cell migration, axon guidance, and neuritogenesis (Gupton et al. 2007; Damiani et al. 2016). Diaph3 knockout mice show re- duced brain size and numbers of PAX6- and TBR2-positive neural progenitors, resulting in altered neocortical neuronal populations (Damiani et al. 2016). In addition, DIAPH3 is required for the formation of filopodia, structures essential for neocortical neurite initiation (Dent et al. 2007; Vorstman et al. 2011). Finally, ZFP804A is localized at synapses and regulates neurite outgrowth and synaptogenesis (Deans et al. 2017). Knockdown of ZNF804A in hNPs alters the expression of cell adhesion genes such as C2ORF80 and STMN3 (Hill et al. 2012) that are involved in neurite outgrowth and axonal and dendritic branching (Poulain et al. 2008). Together, these findings demonstrate that downstream targets of Foxp1 play an essential role in neurogenesis, neuronal migration, and synaptogenesis during brain development, consistent with the phenotypes observed in Foxp1 cKO mice. However, based on the many significantly enriched GO categories (e.g., neuron projection, cytoskeleton organization, and synapse) (Supplemental Table S3), it is also possible that Foxp1 is important for regulating additional cellular phenotypes, such as axonal and/or dendritic outgrowth and/or plasticity. Future studies that investigate the specific downstream targets of Foxp1 within the developing neocortex should provide deeper insights into the cellular phenotypes regulated by Foxp1.

Overlapping the Foxp1 DEGs with lists of genes relevant to ASD (SFARI genes), as we did in Figure 3, can begin to provide translational relevance to the pathways identified downstream from Foxp1 in the mouse brain. In addition to the SFARI list of ASD genes, we also examined the overlap of Foxp1 DEGs with a smaller list of genes (65 genes) that are high-confidence ASD genes with de novo mutations (Sanders et al. 2015). FOXP1 is among this list, and all of these 65 genes are also contained within the SFARI gene list. Ten genes overlap between the Foxp1 DEGs and the high-confidence ASD genes (AKAP9, CHD2, CTTNBP2, ERBIN, FOXP1, GRIN2B, INTS6, KMT2C, SCN2A, and $S H A N K 3)$. This suggests that most of the Foxp1-regulated genes that are associated with ASD are not among the high-confidence genes with de novo mutations. However, since FOXP1 itself is among those genes and is a transcription factor, FOXP1 could be a key upstream regulator of pathways at risk in ASD. Interestingly, a recent report suggested that patients with mutations in FOXP1 have a consistent clinical phenotype with ID and frequent ASD comorbidity (Meerschaut et al. 2017).

Given that Foxp1 cKO mice have altered USVs and structural brain abnormalities, we were surprised that the mice did not have reduced body weight (Fig. 1C). These results suggest that the mutant mice are still cared for by dams and are able to properly feed. Future studies that investigate the long-term impact of developmental loss of Foxp1 in the forebrain should determine whether these mice ultimately have growth or metabolism deficits; however, adult Foxp1 cKO mice do not weigh significantly different from control littermates (Araujo et al. 2017). Additionally, we have not explored the potential impact of loss of Foxp1 in the developing hippocampus. However, we demonstrated recently that hippocampal expression of Foxp1 plays a role in spatial learning and CA1- 
dependent LTP maintenance using the same Foxp1 cKO mice at later stages (Araujo et al. 2017).

\section{Sumoylation of Foxp1 regulates its function in neurons}

Sumoylation is a post-translational modification that regulates transcription, chromatin remodeling, synapse formation, and mitochondrial function in the CNS (Martin et al. 2007; Wilkinson et al. 2010; Hendriks and Vertegaal 2016). Recently, three different groups have reported that sumoylation of FOXP2 regulates gene expression (Estruch et al. 2016; Meredith et al. 2016; Usui et al. 2017), neuronal differentiation, motor function, and vocal communication (Usui et al. 2017). In addition, recent work has demonstrated that rat Foxp1 is sumoylated at K670 in an activity-dependent manner in primary rat neocortical cultures, and this sumoylation modulates Foxp1 function in terms of dendritic morphogenesis and regulation of Cntnap2 expression via CTBP complex recruitment in this model system (Rocca et al. 2017). Here, we demonstrate that sumoylation of Foxp1 regulates neuronal differentiation and migration. We provide biochemical evidence that sumoylation of FOXP1 at K636 (equivalent to rat $\mathrm{K} 670$ ) is regulated by SUMO-1, SUMO-2, and PIAS2/3 (Fig. 4; Supplemental Fig. S3). We also demonstrate that sumoylation switches FOXP1-NuRD complex recruitment (Fig. 6). HDAC1 and HDAC2 are major components of the NuRD complex, which controls neuronal proliferation and specification in neural precursors (Montgomery et al. 2009). HDAC2 also regulates radial glia migration at the embryonic stage (Nott et al. 2013). A recent study has also shown that the NuRD subunits CHD3, $\mathrm{CHD} 4$, and CHD5 regulate layer specification, neural progenitor proliferation, and neuronal migration in the mouse neocortex (Nitarska et al. 2016). Thus, our data suggest future studies to investigate whether sumoylation of FOXP1 controls neocortical development through the regulation of gene expression that is modified via FOXP1-NuRD complex recruitment.

The functional similarities between FOXP2 and FOXP1 in terms of sumoylation indicate that this post-translational modification plays an important role during overall brain development. Sumoylation might therefore also have an important role in ASD pathogenesis. Copy number variations in $S A E 1$, an E1 SUMO ligase for SUMO-1/ $2 / 3$, have been identified in an ASD cohort (Prasad et al. 2012). Therefore, delineating regulation of sumoylation could provide additional insights into the role of FOXP1 during brain development and how alterations to FOXP1 might put individuals at risk for ASD. Further understanding of the role of sumoylation in the brain in general should give rise to novel insights and targets for understanding the molecular mechanisms underlying developmental disorders such as ASD.

\section{Materials and methods}

Constructs and site-directed mutagenesis

Sources of the DNA constructs used in this study were as follows: pLUGIP-FOXP1-3xFlag (generated by cloning), pLUGIP-FOXP1
K636R-3xFlag (generated by mutagenesis as described below), pLUGIP-FOXP2-3xFlag (Konopka et al. 2009), pLUGIP-FOXP2 K674R-3xFlag (Usui et al. 2017), pEGFP-N1-SUMO-1 and pEGFP-N1-SUMO-2 (Rabellino et al. 2012), pLenti6.4-PIAS3-V5 and pLenti6.4-FOXP4-V5 (generated by Invitrogen Gateway system from pENTRD purchased from Open Biosystems), and pLenti6.4-FOXP1 K636R-V5 (generated by cloning). Mutagenesis of pLUGIP-FOXP1 K636R-3xFlag was carried out using the QuikChange II XL site-directed mutagenesis kit (Agilent, 200521) according to the manufacturer's instruction using the following primers: FOXP1 K636R (lysine to arginine): F-5'-GTGCATCCTGT ACACGTCAGAGAAGAGCCCCTCGATCCAG- $3^{\prime}$ and R-5'-CTG GATCGAGGGGCTCTTCTCTGACGTGTACAGGATGCAC-3'

\section{Antibodies}

The following antibodies were used: mouse monoclonal antiSUMO-1 (D-11) antibody (Santa Cruz Biotechnology, sc-5308), rabbit polyclonal anti-FOXP1 antibody (Spiteri et al. 2007), mouse monoclonal anti-FOXP1 (JC12) antibody (Abcam, ab32010), goat polyclonal anti-FOXP2 (N-16) antibody (Santa Cruz Biotechnology, sc-21068), mouse monoclonal anti-Flag M2 antibody (Sigma-Aldrich, F1804), mouse monoclonal antiV5 antibody (Invitrogen, R960-25), goat polyclonal anti-GFP antibody (Rockland Immunochemicals, 600-101-215), chick polyclonal anti-GFP antibody (Aves Laboratories, GFP-1010), rabbit monoclonal anti-SUMO-2/3 (18H8) antibody (Cell Signaling Technology, 4971), rabbit polyclonal anti-PIAS2 antibody (Abcam, ab155556), rabbit polyclonal anti-PIAS3 (H-169) antibody (Santa Cruz Biotechnology, sc-14017), rabbit polyclonal anti-MAP2 antibody (Chemicon, AB5622), mouse monoclonal anti-CtBP (E-12) antibody (Santa Cruz Biotechnology, sc-17759), rabbit polyclonal anti-CDP (CUX1: M-222) antibody (Santa Cruz Biotechnology, sc-13024), rat anti-CTIP2 (Abcam, ab18465), rabbit polyclonal anti-HDAC1 antibody (Abcam, ab19845), mouse monoclonal anti-HDAC1 (10E2) antibody (Cell Signaling Technology, 5256), mouse monoclonal antiHDAC2 (3F3) antibody (Cell Signaling Technology, 5113), rabbit monoclonal anti-MTA1 (D40D1) XP antibody (Cell Signaling Technology, 5647), rabbit polyclonal anti-MTA2 (H-170) antibody (Santa Cruz Biotechnology, sc-28731), rabbit polyclonal anti-p66 $\beta$ (GATAD2B) antibody (Novus Biologicals, NBP187358), mouse monoclonal anti-glyceraldehyde-3-phosphate dehydrogenase (GAPDH) antibody (Millipore, MAB374), mouse (G3A1) mAb IgG1 isotype control (Cell Signaling Technology, 5415), normal rabbit IgG (Cell Signaling Technology, 2729), and normal goat IgG (Santa Cruz Biotechnology, sc-2028). Dilutions of antibodies are described individually in each method.

Mice

Wild-type C57BL/6J, Emx1-Cre (purchased from Jackson Laboratory, 005628) (Gorski et al. 2002), and Foxp1 flox/flox (Feng et al. 2010) mice were used as described previously (Araujo et al. 2017). Emx1-Cre and Foxp $1^{\text {flox/flox }}$ mice were backcrossed with $\mathrm{C} 57 \mathrm{BL} / 6 \mathrm{~J}$ mice for at least 10 generations to obtain congenic animals. PCR genotyping was performed using the following primers: for the Emx1-Cre oIMR1084_mutant, F-5'-GCGGTCTGG CAGTAAAAACTATC-3'; for the oIMR1085_mutant, R-5'-GT GAAACAGCATTGCTGTCACTT-3'; for oIMR4170_wildtype, F-5'-AAGGTGTGGTTCCAGAATCG-3'; and for oIMR4171 wildtype, R-5'-CTCTCCACCAGAAGGCTGAG-3' to detect mutant (102-base-pair [bp]) and wild-type (378-bp) alleles and for flox Foxp1, F-5'-CCAGGGATCAGAGATTACTGTAGC-3' and R-5'-CACCCTCTCCAAGTCTGCCTCAG-3' (Feng et al. 
2010) to detect mutant (280-bp) and wild-type (370-bp) alleles. For timed embryo staging, the day of detection of the vaginal plug was considered to be E0.5. All procedures were approved by the Institutional Animal Care and Use Committee (IACUC) of University of Texas Southwestern Medical Center.

\section{Western blotting}

Proteins from each sample were separated by SDS-PAGE and transferred to an Immun-Blot PVDF membrane (Bio-Rad Laboratories, $162-0177)$, blocked with blocking buffer (1\% skim milk in TBS with $0.1 \%$ Tween-20) for $30 \mathrm{~min}$ at room temperature, and incubated with the following primary antibodies overnight at $4^{\circ} \mathrm{C}$ : mouse anti-Flag M2 $(1: 10,000)$, mouse anti-SUMO-1 (1:100-1000), rabbit anti-FOXP1 (1:2500), goat anti-FOXP2 (1:1000), mouse anti-V5 (1:1000), goat anti-GFP (1:1000), mouse anti-GAPDH (1:5000), mouse anti-CtBP antibody (1:1000), rabbit anti-HDAC1 (1:1000), mouse anti-HDAC1 (1:1000), mouse antiHDAC2 (1:1000), rabbit anti-MTA1 (1:1000), rabbit anti-MTA2 (1:1000), and rabbit anti-GATAD2B (1:400). The membrane was washed with TBS-T (TBS with $0.1 \%$ Tween-20), blocked again, reacted with the appropriate horseradish peroxidase (HRP)-conjugated species-specific secondary antibodies (goat anti-mouse IgG, 1:5000 [Thermo Fisher Scientific, 12-349] and donkey antigoat IgG, 1:5000 [Millipore, AP180P]) or the appropriate speciesspecific secondary antibodies fluorescent dye-conjugated to IRDye 680RD and/or IRDye 800CW (1:20,000; LI-COR Biosciences) according to the manufacturer's instruction for $1 \mathrm{~h}$ at room temperature, washed, and developed using SuperSignal West Pico chemiluminescent substrate (Thermo Fisher Scientific, PI34080) when HRP secondary antibodies were used. The images were collected using Blue Basic Autorad film (BioExpress, F9023-8 $\times 10$ ) or the Odyssey infrared imaging system (LI-COR Biosciences).

\section{USVS}

USV analysis was performed as described previously (Usui et al. 2017). Assessment of USVs was carried out at P4 and P7. Differences between genotypes on all measured features of vocalization were assessed using two-way analysis of variance, testing for the main effects of genotype, day, and interaction of genotype by day. Post hoc multiple comparisons were assessed using Tukey's procedure. Features of vocalization were considered independently.

\section{MRI}

Mice were anesthetized and intracardially perfused (rate $1 \mathrm{~mL}$ / $\mathrm{min}$ ) with $10 \mathrm{~mL}$ of $0.1 \mathrm{M}$ PBS without $\mathrm{Ca}$ and $\mathrm{Mg}$ containing $10 \mathrm{U} / \mathrm{mL}$ heparin (Sagent Pharmaceuticals, 400-30) and $2 \mathrm{mM}$ ProHance (Bracco Diagnostics, 11181) for postnatal pups followed by $15 \mathrm{~mL}$ of $4 \%$ paraformaldehyde (PFA) containing 2 $\mathrm{mM}$ ProHance (a gadolinium contrast agent; Bracco Diagnostics, Inc.) (Spring et al. 2007). The brains and remaining skull structures were incubated in 4\% PFA and $2 \mathrm{mM}$ ProHance overnight at $4^{\circ} \mathrm{C}$ and then transferred to $0.1 \mathrm{M}$ PBS containing $2 \mathrm{mM}$ ProHance and $0.02 \%$ sodium azide for at least 1 mo prior to MRI scanning (de Guzman et al. 2016). A multichannel 7.0 Tesla MRI scanner (Agilent) was used to image the brains within the skulls. Sixteen custom-built solenoid coils were used to image the brains in parallel (Bock et al. 2005; Lerch et al. 2011). In order to get the contrast required for image registration at P7, a threedimensional diffusion weighted fast spin-echo sequence with an echo train length of 6 was used (Sussman et al. 2013), with a TR of $270 \mathrm{msec}$, first TE of $30 \mathrm{msec}$, and TE of $10 \mathrm{msec}$ for the re- maining five echoes; two averages; field of view $14 \times 14 \times 25$ $\mathrm{mm}^{3}$; and a matrix size of $250 \times 250 \times 450$, yielding an image with $0.056-\mathrm{mm}$ isotropic voxels. Diffusion parameters were as follows: $\delta=5 \mathrm{msec}, \Delta=15 \mathrm{msec}, \mathrm{G}=30 \mathrm{G} / \mathrm{cm}$, six high- $b$-value images (using the Tetra 6 partition of the Jones30 diffusion table), yielding a high $b$-value of $2147 \mathrm{sec} / \mathrm{mm}^{2}$. This was done to acquire an improved contrast at the young age. Total imaging time was $14 \mathrm{~h}$. The high- $b$-value images were averaged to create a single diffusion weighted image that had the contrast required for the image registration. To visualize and compare any changes in the mouse brains, the images were linearly (six parameters followed by 12 parameters) and nonlinearly registered together. All scans were then resampled with the appropriate transform and averaged to create a population atlas representing the average anatomy of the study sample. The result of the registration was to have all scans deformed into alignment with each other in an unbiased fashion. This allowed for the analysis of the deformations needed to take each individual mouse's anatomy into this final atlas space, the goal being to model how the deformation fields relate to genotype (Nieman et al. 2006; Lerch et al. 2008). The Jacobian determinants of the deformation fields were then calculated as measures of volume at each voxel. Significant volume changes could then be calculated by warping a pre-existing classified MRI atlas onto the population atlas, which allowed for the volume of 59 segmented structures encompassing neocortical lobes, large white matter structures (i.e., corpus callosum), ventricles, the cerebellum, the brain stem, and olfactory bulbs (Dorr et al. 2008) to be assessed in all brains. Furthermore, these measurements could be examined on a voxel-wise basis in order to localize the differences found within regions or across the brain. Multiple comparisons in this study were controlled for using the false discovery rate (FDR) (Genovese et al. 2002).

Immunohistochemistry and neocortical layer analysis

Immunohistochemistry was performed as described previously (Usui et al. 2012). For CTIP2 staining, antigen retrieval pretreatment was performed by incubating sections in citrate buffer (10 $\mathrm{mM}$ citrate, $0.05 \%$ Tween- 20 at $\mathrm{pH}$ 6) for $10 \mathrm{~min}$ at $95^{\circ} \mathrm{C}$. Sections were stained with the following primary antibodies: rabbit anti-FOXP1 (1:1000), mouse anti-FOXP1 (1:500), goat antiFOXP2 (1:500), mouse anti-SUMO-1 (1:100), rabbit anti-SUMO2/3 (1:200), rabbit anti-PIAS2 (1:200), rabbit anti-PIAS3 (1:100), goat anti-GFP (1:1000), chicken anti-GFP (1:1000), rabbit antiCUX1 (1:100), and rat anti-CTIP2 (1:500). For fluorescence immunostaining, species-specific antibodies conjugated to Alexa fluor 488, Alexa fluor 555, and/or Alexa fluor 647 (1:2000; Invitrogen) were applied, and coverglasses were mounted with ProLong Gold or ProLong Diamond anti-fade mountant with DAPI (Thermo Fisher Scientific, P-36931 or P36971) for nuclear staining. TO-PRO-3 was also used to stain the nucleus. Images were collected using a Zeiss microscope and digital camera system (Axio Observer.Z1, Carl Zeiss) and a Zeiss confocal laser scanning microscope (LSM510 with META or LSM710, Carl Zeiss). Analysis of neocortical layering at P7 was performed using the Fiji image processing package. To measure neocortical layer thickness, coronal sections containing the $\mathrm{S} 1$ cortex were stained for DAPI (all layers), CUX1 (layers 2-4), CTIP2 (layer 5), and Foxp2 (layer 6). For each animal, three measurements spanning the width of the S1 cortex were collected and averaged as that animal's absolute neocortical thickness. Next, for each neocortical layer marker, three measurements along the layer width within S1 were collected and averaged as absolute layer thickness. For each animal, relative layer thickness was calculated as absolute layer thickness divided by absolute neocortical thickness. For 
cell count analysis, numbers of nuclei positive for each layer marker were counted in a $1024 \times 4096$-pixel $(0.36 \times 1.43-\mathrm{mm})$ field of view over S1. Differences between genotypes were assessed using a two-tailed $t$-test ( $n=4-5$ per condition).

\section{RNA-seq}

RNA-seq was performed as a service by BGI America for P0 brains or as described previously (Takahashi et al. 2015) for P7 brains. Briefly, total RNA was extracted with the miRNeasy minikit (Qiagen, 217004) according to the manufacturer's instructions. RNA integrity number (RIN) of total RNA was quantified by an Agilent 2100 Bioanalyzer using an Agilent RNA 6000 nanokit (Agilent, 5067-1511). Total RNA with RIN values of $\geq 9.5$ were used for RNA-seq library preparation. mRNA was purified from $2 \mu \mathrm{g}$ of total RNA by NEXTflex poly(A) beads (Bioo Scientific, 512981), subjected to fragmentation and first and second strand syntheses, and cleaned up by EpiNext beads (EpiGentek, P1063). Second strand DNA was adenylated, ligated, and cleaned up twice by EpiNext beads. cDNA libraries were amplified by PCR and cleaned up twice by EpiNext beads. cDNA library quality was quantified by a 2100 Bioanalyzer using an Agilent highsensitivity DNA kit (Agilent, 5067-4626, ). Libraries of P0 and P7 brains were sequenced separately as 50 -bp single ends on a BGISEQ-500 or BGI in-house sequencer or as 75 -bp single ends on Illumina NextSeq 500, respectively.

\section{RNA-seq data analysis}

Raw reads were first filtered for quality and trimmed for adapters using FASQC (http://www.bioinformatics.babraham.ac.uk/ projects/fastqc) and Trimmomatic (Bolger et al. 2014). Filtered reads were then aligned to the mouse genome mm10 (https:// genome.ucsc.edu) using STAR version 2.5.2b (Dobin et al. 2013), allowing three mismatches. Uniquely mapped reads (bam flag NH:i:1) were used to obtain the gene counts using the HTSeq package (Anders et al. 2015), and the read counts were normalized to the CPM (counts per million) implemented in the edgeR package (Robinson et al. 2010; McCarthy et al. 2012). For further analysis, we performed a sample-specific CPM filtering considering genes with CPM values of 1 in all replicates for treatments or controls. DESeq (Anders and Huber 2010; Love et al. 2014) was then used to detect the DEGs. We applied a filter of FDR (adjusted $P$ value) of $<0.05$ and absolute $\log _{2}$ fold change of $>0.3$ to identify significantly changed genes. GO analysis with the significant DEGs was carried out using ToppGene (https://toppgene.cchmc .org), and these GO terms were consolidated using REVIGO (reduce and visualize GO) (Supek et al. 2011). The list of ASD genes was derived from the SFARI gene database (843 genes) (https:// gene.sfari.org/database/human-gene).

\section{Network analysis}

The R package for WGCNA (Langfelder and Horvath 2008) was used to build a gene coexpression network using filtered CPM data (as described above). A signed network was constructed using the blockwiseModules function of the WGCNA R package. $A$ value of 18 was chosen as $\beta$. For other parameters, we used corType $=$ pearson, maxBlockSize $=15000$, reassignThreshold $=1 \times$ $10^{-4}$, mergeCutHeight $=0.15$, deepSplit $=4$, detectCutHeight $=$ 0.999 , etc. Network plots were created using Cytoscape version 3.4.0 (Shannon et al. 2003), representing the top 200 edges based on ranked weights.

\section{Cell culture}

293T cells (American Type Culture Collection) were cultured in DMEM containing $10 \%$ fetal bovine serum (FBS) (Invitrogen, 10437028) and antibiotic-anti-mycotic (Invitrogen, 15240-062) at $37^{\circ} \mathrm{C}$ under $5 \% \mathrm{CO}_{2}$. Forty-eight hours after transfection using FuGENE6 transfection reagent (Promega, E2691), cells were harvested. Cells were washed with PBS, lysed with a lysis buffer $(20$ $\mathrm{mM}$ Tris- $\mathrm{HCl}$ at $\mathrm{pH}$ 8.0, $1 \mathrm{mM}$ EDTA, $150 \mathrm{mM} \mathrm{NaCl}, 0.1 \%$ [w/v] Triton X-100) with protease inhibitor cocktail (Sigma-Aldrich, P8340) and $50 \mathrm{mM}$ NEM as a sumo protease inhibitor (Sigma-Aldrich, 3876), and processed for sumoylation analysis. $\mathrm{H}_{2} \mathrm{O}_{2}(1$ $\mathrm{mM})$, which reduces the level of high-molecular-weight SUMO conjugates, was used to reduce sumoylated bands for $1 \mathrm{~h}$ before harvesting cells (Bossis and Melchior 2006). Ginkgolic acid (100 $\mu \mathrm{M}$; Sigma-Aldrich, 75741) was also used to reduce sumoylated bands as a sumoylation-specific inhibitor for $6 \mathrm{~h}$ before harvesting cells (Fukuda et al. 2009; Meredith et al. 2016).

$m N P S$

mNPs were cultured from E13.5 neocortices as described previously (Usui et al. 2017). Cells were expanded for $2 \mathrm{~d}$ before transduction by lentivirus. After transduction, cells were cultured in neurobasal medium (Invitrogen, 21103-049A) supplemented with B27 and $500 \mu \mathrm{M}$ L-glutamine (Invitrogen, 25030081) to induce neuronal differentiation. Culture medium was not changed after transduction.

$h N P s$

hNPs (Lonza, PT-2599) were cultured as described previously (Konopka et al. 2012; Usui et al. 2017). Lentiviral transduction was used to introduce genes. Forty-eight hours after transduction, cells were collected with QIAzol lysis reagent (Qiagen, 79306) and processed for total RNA purification.

\section{Human neurospheres}

To make neurospheres of hNPs, proliferating hNPs were plated at a density of 300,000 cells per well in neurobasal A medium supplemented with BIT, antibiotic-anti-mycotic, glutamax, $10 \mathrm{ng} /$ $\mathrm{mL}$ FGF, and $10 \mathrm{ng} / \mathrm{mL}$ EGF on Corning spheroid 96-well black/ clear round-bottomed, ultra-low-attachment surface, lidded sterile microplates (Sigma-Aldrich, CLS4520) and incubated at $37^{\circ} \mathrm{C}$ under $5 \% \mathrm{CO}_{2}$. Cells were half-fed every second day to maintain the concentration of growth factors. To assess neurosphere migration, proliferating hNPs were plated at a density of 300,000 cells per well in neurobasal A medium supplemented with BIT, antibiotic-anti-mycotic glutamax, $10 \mathrm{ng} / \mathrm{mL}$ FGF, and $10 \mathrm{ng} / \mathrm{mL}$ EGF on Corning spheroid 96-well black/clear round-bottomed, ultralow-attachment surface, lidded sterile microplates; infected with lentivirus at the same time; and incubated at $37^{\circ} \mathrm{C}$ under $5 \% \mathrm{CO}_{2}$. Forty-eight hours after plating and lentiviral infection, culture medium was replaced with neurobasal A medium supplemented with B27 minus vitamin A, antibiotic-anti-mycotic glutamax, $10 \mathrm{ng} / \mathrm{mL} \mathrm{BDNF}$, and $10 \mathrm{ng} / \mathrm{mL} \mathrm{NT}-3$, and then neurospheres were gently transferred on poly-L-ornithine- and laminin-coated plates using Axygen 200- $\mu \mathrm{L}$ wide-bore tips (Corning, TF-205-WB-R-S). Twenty-four hours after plating, neurospheres were fixed, stained, and quantified.

\section{Lentivirus production}

Lentivirus production was performed as described previously (Usui et al. 2017). 
Usui et al.

\section{Immunoprecipitation}

Immunoprecipitation was performed as described previously (Usui et al. 2017). The following amounts of antibodies were used: $10 \mu \mathrm{L}$ of mouse anti-SUMO-1 per immunoprecipitation, $10 \mu \mathrm{L}$ of rabbit anti-FOXP1 per immunoprecipitation, $5 \mu \mathrm{L}$ of mouse anti-Flag M2 per immunoprecipitation, $2 \mu \mathrm{L}$ of mouse anti-V5 per immunoprecipitation, and $10 \mu \mathrm{L}$ of goat anti-FOXP2 per immunoprecipitation. Mouse, rabbit, and goat IgGs were used as appropriate negative controls.

\section{Immunocytochemistry}

Cultured cells were fixed with 4\% PFA in PBS for $10 \mathrm{~min}$ at room temperature, placed in PBS, and then permeabilized in PBS-T. Blocking was performed using $10 \%$ goat serum and $1 \%$ BSA in PBS-T for $1 \mathrm{~h}$ at room temperature. Cells were incubated with the following primary antibodies overnight at $4^{\circ} \mathrm{C}$ : goat antiGFP $(1: 1,000)$, mouse anti-Flag M2 $(1: 1,000)$, and rabbit antiMAP2 $(1: 1,000)$. Cells were then washed and incubated with secondary antibodies for $1 \mathrm{~h}$ at room temperature. For fluorescence immunostaining, species-specific antibodies conjugated to Alexa fluor 488, Alexa fluor 555, and/or Alexa fluor 647 (1:2000; Invitrogen) were applied, and coverslips were mounted with ProLong Gold anti-fade mountant containing DAPI for nuclear staining (Invitrogen, P36934). TO-PRO-3 (Invitrogen, T3605) was also used to stain the nucleus. Images were collected using a Zeiss microscope and digital camera system (Axio Observer.Z1, Zeiss) and Zeiss confocal laser scanning microscope (LSM 510 with META, Zeiss).

IUE

IUE was performed as described previously (Usui et al. 2017). Timed pregnant mice were deeply anesthetized with isoflurane $(1.5 \%-3 \%$ in oxygen) during surgery and were also administered $1 \mathrm{mg} / \mathrm{kg}$ buprenorphine to limit pain after electroporation. Endotoxin-free plasmid DNA $(1-2 \mu \mathrm{g} / \mu \mathrm{L})$ with $0.1 \%$ Fast Green FCF dye (Sigma-Aldrich, F7252) was microinjected into the lateral ventricles of E14.5 embryos to target upper layer (layer 2/3) neurons of the neocortex. The embryo was held through the uterus with platinum plate electrode tweezers (Protech International, Inc., CUY650P5) and electroporated (five 50-msec pulses of 33 $\mathrm{V}$ with an interval of $950 \mathrm{msec}$ ) (NEPA Gene, CUY21SC) (Baek et al. 2015; Li et al. 2015). Electroporated embryos were analyzed at E18.5.

\section{Statistical analysis}

All data are represented as means of biological independent experiments \pm SEM. Statistical analysis ( $t$-test, one-way ANOVA, or two-way ANOVA with a Tukey's multiple comparison test) was performed using Prism 7. Asterisks indicate $P$-values $(P<$ $0.001\left[{ }^{* * *}\right], P<0.01\left[{ }^{* *}\right]$, and $\left.P<0.05\left[{ }^{*}\right]\right)$.

Accession numbers

The NCBI Gene Expression Omnibus accession number for the RNA-seq data reported in this study is GSE98913.

\section{Acknowledgments}

We thank Dr. Haley O. Tucker for providing the Foxp $1^{\text {flox/flox }}$ mice, and the Neuroscience Microscopy Facility at University of Texas Southwestern for imaging support. N.U. is a Research
Fellow of the Uehara Memorial Foundation. M.C. is a Predoctoral Fellow of the Autism Science Foundation. G.K. is a Jon Heighten Scholar in Autism Research at University of Texas Southwestern. This work was supported by the Japan Society for the Promotion of Science Program for Advancing Strategic International Networks to Accelerate the Circulation of Talented Researchers (S2603 to N.U., K.T., and G.K.); a National Science Foundation Graduate Research Fellowship Program grant (2013162469 to D.J.A.); the Howard Hughes Medical Institute Med into Grad Initiative and the National Institutes of Health $\left(\mathrm{NIH}_{\text {; TL1TR001104 }}\right.$ and T32GM109776 to M.C.); the Ontario Brain Institute, Brain Canada, and the Canadian Institute for Health Research (to J.P.L.); and a grant from the Simons Foundation (SFARI 401220), a research grant from the March of Dimes Foundation (5-FY1319), and grants from the NIH (DC014702 and MH102603) (to G.K.). N.U. and G.K. designed the study, analyzed the data, and wrote the paper. N.U., D.J.A., M.C., M.H., and K.T. performed experiments and analyses. A.K. performed bioinformatic analyses. J.E. and J.P.L. performed MRI analyses. G.K. supervised this study and provided intellectual guidance. All authors discussed the results and commented on the manuscript.

\section{References}

Anders S, Huber W. 2010. Differential expression analysis for sequence count data. Genome Biol 11: R106.

Anders S, Pyl PT, Huber W. 2015. HTSeq-a Python framework to work with high-throughput sequencing data. Bioinformatics 31: 166-169.

Araujo DI, Anderson AG, Berto S, Runnels W, Harper M, Ammanuel S, Rieger MA, Huang HC, Rajkovich K, Loerwald KW, et al. 2015. FoxP1 orchestration of ASD-relevant signaling pathways in the striatum. Genes Dev 29: 2081-2096.

Araujo DJ, Toriumi K, Escamilla CO, Kulkarni A, Anderson AG, Harper M, Usui N, Ellegood J, Lerch JP, Birnbaum SG, et al. 2017. Foxp1 in forebrain pyramidal neurons controls gene expression required for spatial learning and synaptic plasticity. I Neurosci 37: 10917-10931.

Arriaga G, Jarvis ED. 2013. Mouse vocal communication system: are ultrasounds learned or innate? BrainLang 124: 96-116.

Arriaga G, Zhou EP, Jarvis ED. 2012. Of mice, birds, and men: the mouse ultrasonic song system has some features similar to humans and song-learning birds. PLoS One 7: e46610.

Bacon C, Rappold GA. 2012. The distinct and overlapping phenotypic spectra of FOXP1 and FOXP2 in cognitive disorders. Hum Genet 131: 1687-1698.

Bacon C, Schneider M, Le Magueresse C, Froehlich H, Sticht C, Gluch C, Monyer H, Rappold GA. 2015. Brain-specific Foxp1 deletion impairs neuronal development and causes autistic-like behaviour. Mol Psychiatry 20: 632-639.

Baek ST, Copeland B, Yun EJ, Kwon SK, Guemez-Gamboa A, Schaffer AE, Kim S, Kang HC, Song S, Mathern GW, et al. 2015. An AKT3-FOXG1-reelin network underlies defective migration in human focal malformations of cortical development. Nat Med 21: 1445-1454.

Basta J, Rauchman M. 2015. The nucleosome remodeling and deacetylase complex in development and disease. Transl Res 165: 36-47.

Berkowicz SR, Featherby TJ, Qu Z, Giousoh A, Borg NA, Heng JI, Whisstock JC, Bird PI. 2016. Brinp1 $1^{-/-}$mice exhibit autismlike behaviour, altered memory, hyperactivity and increased parvalbumin-positive cortical interneuron density. Mol Autism 7: 22. 
Bock NA, Nieman BJ, Bishop JB, Mark Henkelman R. 2005. In vivo multiple-mouse MRI at 7 Tesla. Magn Reson Med 54: 1311-1316.

Bolger AM, Lohse M, Usadel B. 2014. Trimmomatic: a flexible trimmer for Illumina sequence data. Bioinformatics 30: 2114-2120.

Bossis G, Melchior F. 2006. Regulation of SUMOylation by reversible oxidation of SUMO conjugating enzymes. Mol Cell 21: 349-357.

Chen T, Dent SY. 2014. Chromatin modifiers and remodellers: regulators of cellular differentiation. Nat Rev Genet 15: 93-106.

Chokas AL, Trivedi CM, Lu MM, Tucker PW, Li S, Epstein JA, Morrisey EE. 2010. Foxp1/2/4-NuRD interactions regulate gene expression and epithelial injury response in the lung via regulation of interleukin-6. $J$ Biol Chem 285: 13304-13313.

Damiani D, Goffinet AM, Alberts A, Tissir F. 2016. Lack of Diaph3 relaxes the spindle checkpoint causing the loss of neural progenitors. Nat Commun 7: 13509.

Deans PJ, Raval P, Sellers KJ, Gatford NJ, Halai S, Duarte RR, Shum C, Warre-Cornish K, Kaplun VE, Cocks G, et al. 2017. Psychosis risk candidate ZNF804A localizes to synapses and regulates neurite formation and dendritic spine structure. Biol Psychiatry 82: 49-61.

de Guzman AE, Wong MD, Gleave JA, Nieman BJ. 2016. Variations in post-perfusion immersion fixation and storage alter MRI measurements of mouse brain morphometry. Neuroimage 142: 687-695.

de la Torre-Ubieta L, Won H, Stein JL, Geschwind DH. 2016. Advancing the understanding of autism disease mechanisms through genetics. Nat Med 22: 345-361.

Delmonte S, Gallagher L, O'Hanlon E, McGrath J, Balsters JH. 2013. Functional and structural connectivity of frontostriatal circuitry in autism spectrum disorder. Front Hum Neurosci 7: 430.

Dent EW, Kwiatkowski AV, Mebane LM, Philippar U, Barzik M, Rubinson DA, Gupton S, Van Veen JE, Furman C, Zhang J, et al. 2007. Filopodia are required for cortical neurite initiation. Nat Cell Biol 9: 1347-1359.

Dobin A, Davis CA, Schlesinger F, Drenkow J, Zaleski C, Jha S, Batut P, Chaisson M, Gingeras TR. 2013. STAR: ultrafast universal RNA-seq aligner. Bioinformatics 29: 15-21.

Dorr AE, Lerch JP, Spring S, Kabani N, Henkelman RM. 2008. High resolution three-dimensional brain atlas using an average magnetic resonance image of 40 adult C57Bl/6J mice. Neuroimage 42: 60-69.

Estruch SB, Graham SA, Deriziotis P, Fisher SE. 2016. The language-related transcription factor FOXP2 is post-translationally modified with small ubiquitin-like modifiers. Sci Rep 6: 20911.

Feng X, Ippolito GC, Tian L, Wiehagen K, Oh S, Sambandam A, Willen J, Bunte RM, Maika SD, Harriss JV, et al. 2010. Foxp1 is an essential transcriptional regulator for the generation of quiescent naive $\mathrm{T}$ cells during thymocyte development. Blood 115: 510-518.

Ferland RJ, Cherry TJ, Preware PO, Morrisey EE, Walsh CA. 2003. Characterization of Foxp2 and Foxp1 mRNA and protein in the developing and mature brain. I Comp Neurol 460: 266-279.

Fisher SE, Scharff C. 2009. FOXP2 as a molecular window into speech and language. Trends Genet 25: 166-177.

Frohlich H, Rafiullah R, Schmitt N, Abele S, Rappold GA. 2017. Foxp1 expression is essential for sex-specific murine neonatal ultrasonic vocalization. Hum Mol Genet 26: 1511-1521.
Fukuda I, Ito A, Hirai G, Nishimura S, Kawasaki H, Saitoh H, Kimura K, Sodeoka M, Yoshida M. 2009. Ginkgolic acid inhibits protein SUMOylation by blocking formation of the E1SUMO intermediate. Chem Biol 16: 133-140.

Geiss-Friedlander R, Melchior F. 2007. Concepts in sumoylation: a decade on. Nat Rev Mol Cell Biol 8: 947-956.

Genovese CR, Lazar NA, Nichols T. 2002. Thresholding of statistical maps in functional neuroimaging using the false discovery rate. Neuroimage 15: 870-878.

Gill G. 2003. Post-translational modification by the small ubiquitin-related modifier SUMO has big effects on transcription factor activity. Curr Opin Genet Dev 13: 108-113.

Gorski JA, Talley T, Qiu M, Puelles L, Rubenstein JL, Jones KR. 2002. Cortical excitatory neurons and glia, but not GABAergic neurons, are produced in the Emxl-expressing lineage. I Neurosci 22: 6309-6314.

Greig LC, Woodworth MB, Galazo MJ, Padmanabhan H, Macklis JD. 2013. Molecular logic of neocortical projection neuron specification, development and diversity. Nat Rev Neurosci 14: 755-769.

Gupton SL, Eisenmann K, Alberts AS, Waterman-Storer CM. 2007. mDia2 regulates actin and focal adhesion dynamics and organization in the lamella for efficient epithelial cell migration. J Cell Sci 120: 3475-3487.

Hamdan FF, Daoud H, Rochefort D, Piton A, Gauthier J, Langlois M, Foomani G, Dobrzeniecka S, Krebs MO, Joober R, et al. 2010. De novo mutations in FOXP1 in cases with intellectual disability, autism, and language impairment. Am J Hum Genet 87: 671-678.

Hammerschmidt K, Whelan G, Eichele G, Fischer J. 2015. Mice lacking the cerebral cortex develop normal song: insights into the foundations of vocal learning. Sci Rep 5: 8808.

Hasegawa Y, Yoshida D, Nakamura Y, Sakakibara S. 2014. Spatiotemporal distribution of SUMOylation components during mouse brain development. J Comp Neurol 522: 3020-3036.

Hendriks IA, Vertegaal AC. 2016. A comprehensive compilation of SUMO proteomics. Nat Rev Mol Cell Biol 17: 581-595.

Hill MJ, Jeffries AR, Dobson RJ, Price J, Bray NJ. 2012. Knockdown of the psychosis susceptibility gene ZNF804A alters expression of genes involved in cell adhesion. Hum Mol Genet 21: $1018-1024$.

Iossifov I, O'Roak BJ, Sanders SJ, Ronemus M, Krumm N, Levy D, Stessman HA, Witherspoon KT, Vives L, Patterson KE, et al. 2014. The contribution of de novo coding mutations to autism spectrum disorder. Nature 515: 216-221.

Kaestner KH, Knochel W, Martinez DE. 2000. Unified nomenclature for the winged helix/forkhead transcription factors. Genes Dev 14: 142-146.

Kalueff AV, Stewart AM, Song C, Berridge KC, Graybiel AM, Fentress JC. 2016. Neurobiology of rodent self-grooming and its value for translational neuroscience. Nat Rev Neurosci 17: 45-59.

Kaminsky EB, Kaul V, Paschall J, Church DM, Bunke B, Kunig D, Moreno-De-Luca D, Moreno-De-Luca A, Mulle JG, Warren ST, et al. 2011. An evidence-based approach to establish the functional and clinical significance of copy number variants in intellectual and developmental disabilities. Genet Med 13: $777-784$.

Kang HI, Kawasawa YI, Cheng F, Zhu Y, Xu X, Li M, Sousa AM, Pletikos M, Meyer KA, Sedmak G, et al. 2011. Spatio-temporal transcriptome of the human brain. Nature 478: 483-489.

Konopka G, Roberts TF. 2016a. Animal models of speech and vocal communication deficits associated with psychiatric disorders. Biol Psychiatry 79: 53-61. 
Konopka G, Roberts TF. 2016b. Insights into the neural and genetic basis of vocal communication. Cell 164: 1269-1276.

Konopka G, Bomar JM, Winden K, Coppola G, Jonsson ZO, Gao F, Peng S, Preuss TM, Wohlschlegel JA, Geschwind DH. 2009. Human-specific transcriptional regulation of CNS development genes by FOXP2. Nature 462: 213-217.

Konopka G, Wexler E, Rosen E, Mukamel Z, Osborn GE, Chen L, Lu D, Gao F, Gao K, Lowe JK, et al. 2012. Modeling the functional genomics of autism using human neurons. Mol Psychiatry 17: 202-214.

Kuo HY, Liu FC. 2017. Valproic acid induces aberrant development of striatal compartments and corticostriatal pathways in a mouse model of autism spectrum disorder. FASEB $J$ 31: 4458-4471.

Langen M, Kas MJ, Staal WG, van Engeland H, Durston S. 2011. The neurobiology of repetitive behavior: of mice. Neurosci Biobehav Rev 35: 345-355.

Langfelder P, Horvath S. 2008. WGCNA: an R package for weighted correlation network analysis. BMC Bioinformatics 9: 559 .

Lerch JP, Carroll JB, Spring S, Bertram LN, Schwab C, Hayden MR, Henkelman RM. 2008. Automated deformation analysis in the YAC128 Huntington disease mouse model. Neuroimage 39: 32-39.

Lerch JP, Sled JG, Henkelman RM. 2011. MRI phenotyping of genetically altered mice. Methods Mol Biol 711: 349-361.

Li S, Weidenfeld J, Morrisey EE. 2004. Transcriptional and DNA binding activity of the Foxp1/2/4 family is modulated by heterotypic and homotypic protein interactions. Mol Cell Biol 24: 809-822.

Li X, Xiao J, Frohlich H, Tu X, Li L, Xu Y, Cao H, Qu J, Rappold GA, Chen JG. 2015. Foxp1 regulates cortical radial migration and neuronal morphogenesis in developing cerebral cortex. PLoS One 10: e0127671.

Love MI, Huber W, Anders S. 2014. Moderated estimation of fold change and dispersion for RNA-seq data with DESeq2. Genome Biol 15: 550.

Lozano R, Vino A, Lozano C, Fisher SE, Deriziotis P. 2015. A de novo FOXP1 variant in a patient with autism, intellectual disability and severe speech and language impairment. Eur $J$ Hum Genet 23: 1702-1707.

Martin S, Wilkinson KA, Nishimune A, Henley JM. 2007. Emerging extranuclear roles of protein SUMOylation in neuronal function and dysfunction. Nat Rev Neurosci 8: 948-959.

McCarthy DJ, Chen Y, Smyth GK. 2012. Differential expression analysis of multifactor RNA-seq experiments with respect to biological variation. Nucleic Acids Res 40: 4288-4297.

Meerschaut I, Rochefort D, Revencu N, Petre J, Corsello C, Rouleau GA, Hamdan FF, Michaud JL, Morton J, Radley J, et al. 2017. FOXP1-related intellectual disability syndrome: a recognisable entity. J Med Genet 54: 613-623.

Meredith LJ, Wang CM, Nascimento L, Liu R, Wang L, Yang WH. 2016. The key regulator for language and speech development, FOXP2, is a novel substrate for SUMOylation. J Cell Biochem 117: 426-438.

Molyneaux BJ, Arlotta P, Menezes JR, Macklis JD. 2007. Neuronal subtype specification in the cerebral cortex. Nat Rev Neurosci 8: 427-437.

Montgomery RL, Hsieh J, Barbosa AC, Richardson JA, Olson EN. 2009. Histone deacetylases 1 and 2 control the progression of neural precursors to neurons during brain development. Proc Natl Acad Sci 106: 7876-7881.

Murugan M, Harward S, Scharff C, Mooney R. 2013. Diminished FoxP2 levels affect dopaminergic modulation of corticostriatal signaling important to song variability. Neuron 80: 1464-1476.
Nieman BJ, Flenniken AM, Adamson SL, Henkelman RM, Sled JG. 2006. Anatomical phenotyping in the brain and skull of a mutant mouse by magnetic resonance imaging and computed tomography. Physiol Genomics 24: 154-162.

Nitarska J, Smith JG, Sherlock WT, Hillege MM, Nott A, Barshop WD, Vashisht AA, Wohlschlegel JA, Mitter R, Riccio A. 2016. A functional switch of NuRD chromatin remodeling complex subunits regulates mouse cortical development. Cell Rep 17: $1683-1698$.

Nott A, Nitarska J, Veenvliet JV, Schacke S, Derijck AA, Sirko P, Muchardt C, Pasterkamp RJ, Smidt MP, Riccio A. 2013. Snitrosylation of HDAC2 regulates the expression of the chromatin-remodeling factor Brm during radial neuron migration. Proc Natl Acad Sci 110: 3113-3118.

O'Roak BJ, Deriziotis P, Lee C, Vives L, Schwartz JJ, Girirajan S, Karakoc E, Mackenzie AP, Ng SB, Baker C, et al. 2011. Exome sequencing in sporadic autism spectrum disorders identifies severe de novo mutations. Nat Genet 43: 585-589.

Packer A. 2016. Neocortical neurogenesis and the etiology of autism spectrum disorder. Neurosci Biobehav Rev 64: 185-195.

Penagarikano O, Abrahams BS, Herman EI, Winden KD, Gdalyahu A, Dong H, Sonnenblick LI, Gruver R, Almajano J, Bragin A, et al. 2011. Absence of CNTNAP2 leads to epilepsy, neuronal migration abnormalities, and core autism-related deficits. Cell 147: 235-246.

Portfors CV, Perkel DJ. 2014. The role of ultrasonic vocalizations in mouse communication. Curr Opin Neurobiol 28: 115-120.

Poulain FE, Chauvin S, Wehrle R, Desclaux M, Mallet J, Vodjdani G, Dusart I, Sobel A. 2008. SCLIP is crucial for the formation and development of the Purkinje cell dendritic arbor. J Neurosci 28: 7387-7398.

Prasad A, Merico D, Thiruvahindrapuram B, Wei J, Lionel AC, Sato D, Rickaby J, Lu C, Szatmari P, Roberts W, et al. 2012. A discovery resource of rare copy number variations in individuals with autism spectrum disorder. G3 2: 1665-1685.

Rabellino A, Carter B, Konstantinidou G, Wu SY, Rimessi A, Byers LA, Heymach JV, Girard L, Chiang CM, Teruya-Feldstein J, et al. 2012. The SUMO E3-ligase PIAS1 regulates the tumor suppressor PML and its oncogenic counterpart PMLRARA. Cancer Res 72: 2275-2284.

Robinson MD, McCarthy DI, Smyth GK. 2010. edgeR: a Bioconductor package for differential expression analysis of digital gene expression data. Bioinformatics 26: 139-140.

Rocca DL, Wilkinson KA, Henley JM. 2017. SUMOylation of FOXP1 regulates transcriptional repression via CtBP1 to drive dendritic morphogenesis. Sci Rep 7: 877.

Sanders SJ, He X, Willsey AJ, Ercan-Sencicek AG, Samocha KE, Cicek AE, Murtha MT, Bal VH, Bishop SL, Dong S, et al. 2015. Insights into autism spectrum disorder genomic architecture and biology from 71 risk loci. Neuron 87: 1215-1233.

Scattoni ML, Crawley J, Ricceri L. 2009. Ultrasonic vocalizations: a tool for behavioural phenotyping of mouse models of neurodevelopmental disorders. Neurosci Biobehav Rev 33: 508-515.

Shannon P, Markiel A, Ozier O, Baliga NS, Wang JT, Ramage D, Amin N, Schwikowski B, Ideker T. 2003. Cytoscape: a software environment for integrated models of biomolecular interaction networks. Genome Res 13: 2498-2504.

Sharrocks AD. 2006. PIAS proteins and transcriptional regulation -more than just SUMO E3 ligases? Genes Dev 20: 754-758.

Shepherd GM. 2013. Corticostriatal connectivity and its role in disease. Nat Rev Neurosci 14: 278-291.

Sia GM, Clem RL, Huganir RL. 2013. The human language-associated gene SRPX2 regulates synapse formation and vocalization in mice. Science 342: 987-991. 
Sollis E, Graham SA, Vino A, Froehlich H, Vreeburg M, Dimitropoulou D, Gilissen C, Pfundt R, Rappold GA, Brunner HG, et al. 2016. Identification and functional characterization of de novo FOXP1 variants provides novel insights into the etiology of neurodevelopmental disorder. Hum Mol Genet 25: $546-557$.

Spiteri E, Konopka G, Coppola G, Bomar J, Oldham M, Ou J, Vernes SC, Fisher SE, Ren B, Geschwind DH. 2007. Identification of the transcriptional targets of FOXP2, a gene linked to speech and language, in developing human brain. Am J Hum Genet 81: 1144-1157.

Spring S, Lerch JP, Henkelman RM. 2007. Sexual dimorphism revealed in the structure of the mouse brain using three-dimensional magnetic resonance imaging. Neuroimage 35: 1424-1433.

Stein JL, de la Torre-Ubieta L, Tian Y, Parikshak NN, Hernandez IA, Marchetto MC, Baker DK, Lu D, Hinman CR, Lowe JK, et al. 2014. A quantitative framework to evaluate modeling of cortical development by neural stem cells. Neuron 83: 69-86.

Stessman HA, Xiong B, Coe BP, Wang T, Hoekzema K, Fenckova M, Kvarnung M, Gerdts J, Trinh S, Cosemans N, et al. 2017. Targeted sequencing identifies 91 neurodevelopmental-disorder risk genes with autism and developmental-disability biases. Nat Genet 49: 515-526.

Supek F, Bosnjak M, Skunca N, Smuc T. 2011. REVIGO summarizes and visualizes long lists of gene ontology terms. PLoS One 6: e21800.

Sussman D, Ellegood J, Henkelman M. 2013. A gestational ketogenic diet alters maternal metabolic status as well as offspring physiological growth and brain structure in the neonatal mouse. BMC Pregnancy Childbirth 13: 198.

Takahashi JS, Kumar V, Nakashe P, Koike N, Huang HC, Green CB, Kim TK. 2015. ChIP-seq and RNA-seq methods to study circadian control of transcription in mammals. Methods Enzymol 551: 285-321.

Talkowski ME, Rosenfeld JA, Blumenthal I, Pillalamarri V, Chiang C, Heilbut A, Ernst C, Hanscom C, Rossin E, Lindgren
AM, et al. 2012. Sequencing chromosomal abnormalities reveals neurodevelopmental loci that confer risk across diagnostic boundaries. Cell 149: 525-537.

Usui N, Watanabe K, Ono K, Tomita K, Tamamaki N, Ikenaka K, Takebayashi H. 2012. Role of motoneuron-derived neurotrophin 3 in survival and axonal projection of sensory neurons during neural circuit formation. Development 139: 1125-1132.

Usui N, Co M, Harper M, Rieger MA, Dougherty JD, Konopka G. 2017. Sumoylation of FOXP2 regulates motor function and vocal communication through Purkinje cell development. Biol Psychiatry 81: 220-230.

Vernes SC, Newbury DF, Abrahams BS, Winchester L, Nicod J, Groszer M, Alarcon M, Oliver PL, Davies KE, Geschwind $\mathrm{DH}$, et al. 2008. A functional genetic link between distinct developmental language disorders. $N$ Engl $I$ Med 359: 2337-2345.

Vorstman JA, van Daalen E, Jalali GR, Schmidt ER, Pasterkamp RJ, de Jonge M, Hennekam EA, Janson E, Staal WG, van der Zwaag B, et al. 2011. A double hit implicates DIAPH3 as an autism risk gene. Mol Psychiatry 16: 442-451.

Weiss LA, Shen Y, Korn JM, Arking DE, Miller DT, Fossdal R, Saemundsen E, Stefansson H, Ferreira MA, Green T, et al. 2008. Association between microdeletion and microduplication at 16p11.2 and autism. $N$ Engl I Med 358: 667-675.

Wilkinson KA, Nakamura Y, Henley JM. 2010. Targets and consequences of protein SUMOylation in neurons. Brain Res Rev 64: 195-212.

Willsey AJ, Sanders SI, Li M, Dong S, Tebbenkamp AT, Muhle RA, Reilly SK, Lin L, Fertuzinhos S, Miller JA, et al. 2013. Coexpression networks implicate human midfetal deep cortical projection neurons in the pathogenesis of autism. Cell 155: 997-1007.

Wilson PM, Fryer RH, Fang Y, Hatten ME. 2010. Astn2, a novel member of the astrotactin gene family, regulates the trafficking of ASTN1 during glial-guided neuronal migration. I Neurosci 30: 8529-8540. 


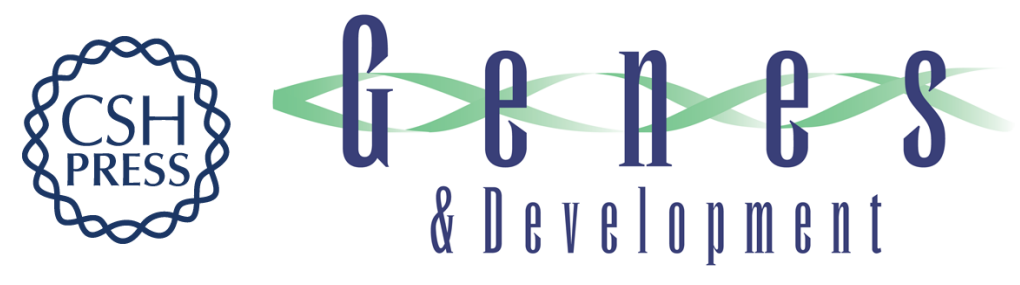

\title{
Foxp1 regulation of neonatal vocalizations via cortical development
}

\author{
Noriyoshi Usui, Daniel J. Araujo, Ashwinikumar Kulkarni, et al.
}

Genes Dev. 2017, 31: originally published online November 14, 2017

Access the most recent version at doi:10.1101/gad.305037.117

\section{Supplemental Material \\ http://genesdev.cshlp.org/content/suppl/2017/11/14/gad.305037.117.DC1 \\ References \\ This article cites 101 articles, 18 of which can be accessed free at: http://genesdev.cshlp.org/content/31/20/2039.full.html\#ref-list-1}

Creative Commons License

Email Alerting Service
This article is distributed exclusively by Cold Spring Harbor Laboratory Press for the first six months after the full-issue publication date (see

http://genesdev.cshlp.org/site/misc/terms.xhtml). After six months, it is available under a Creative Commons License (Attribution-NonCommercial 4.0 International), as described at http://creativecommons.org/licenses/by-nc/4.0/.

Receive free email alerts when new articles cite this article - sign up in the box at the top right corner of the article or click here.

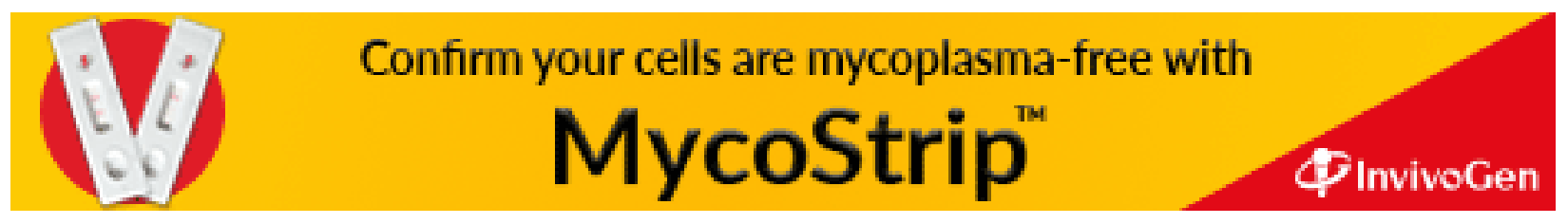

